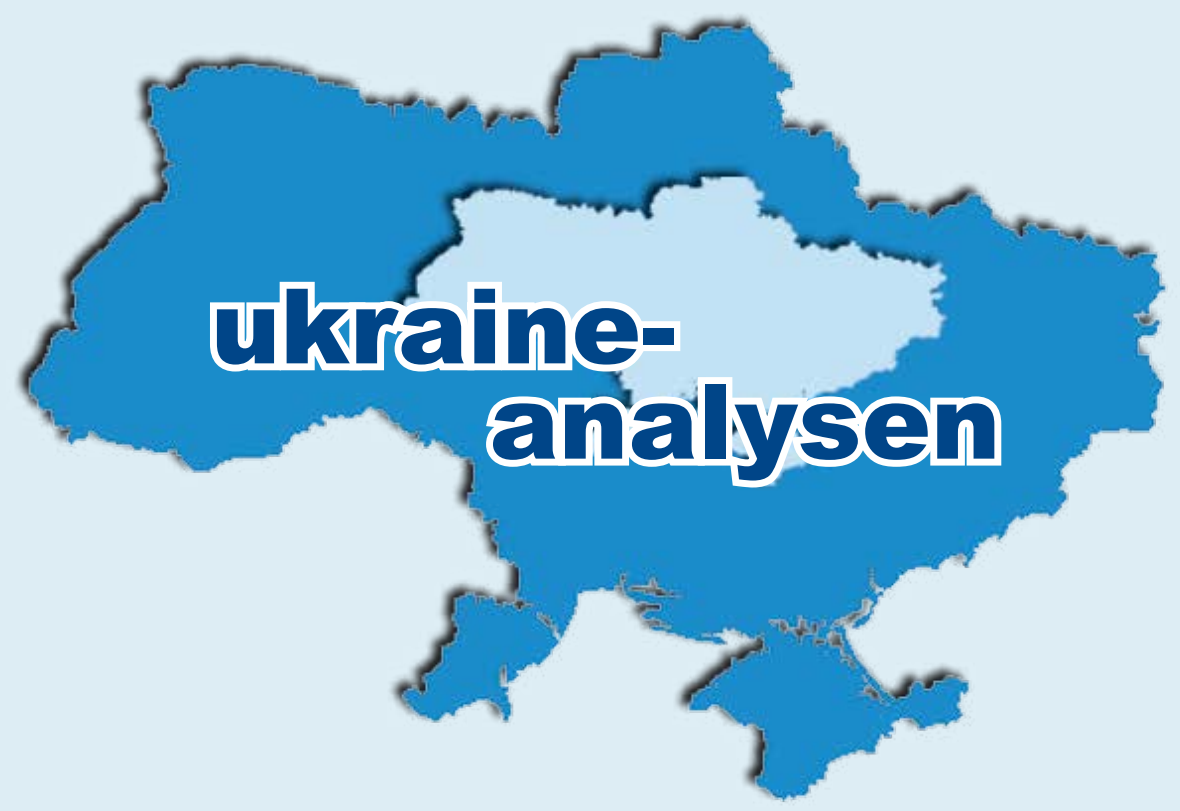

www.laender-analysen.de/ukraine

\title{
VERFASSUNGSKRISE SICHERHEITSDIENST
}

ANALYSE

Das ukrainische Verfassungsgericht kippt Teile der Antikorruptionsreform in der Ukraine

Von Andrii Nekoliak (Universität Tartu)

DOKUMENTATION

Chronologie der Verfassungskrise, 27. Oktober - 06. November 2020

Die Richter des Verfassungsgerichts und ihr Abstimmungsverhalten zur Antikorruptionsreform

- UMFRAGEN

Vertrauen in die Justiz

- DOKUMENTATION

Erklärung zivilgesellschaftlicher Organisationen zur Entscheidung des Verfassungsgerichts

- ANALYSE

Instabiles Vertrauen: Warum Reformen die Glaubwürdigkeit des Geheimdiensts SBU bei der Öffentlichkeit kaum gestärkt haben

Von Eli C. Kaul (Kent State University)

UMFRAGEN

Vertrauen in den Sicherheitsdienst

- STATISTIK

Personalstärke des SBU

- STATISTIK

Die Verbreitung von Covid-19 in der Ukraine

CHRONIK

Covid-19-Chronik, 9. - 22. November 2020

CHRONIK

9. -22 . November 2020

DEUTSCHES
POLEN
INSTITUT

Forschungsstelle Osteuropa

an der Universität Bremen

- Forschungsstelle Osteuropa an der Universität Bremen
Leibniz-Institut fü

Agrarentwicklung in Transformationsökonomien

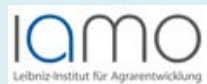

Leibniz-Institut für st- und Südosteuropaforschung
Zentrum für Osteuropa- und internationale Studien (ZOiS) $\mathrm{gGmbH}$ 


\section{Das ukrainische Verfassungsgericht kippt Teile der Antikorruptionsreform in der Ukraine}

Von Andrii Nekoliak (Universität Tartu)

DOI: 10.31205/UA.243.01

\section{Zusammenfassung}

Am 27. Oktober 2020 verkündete das ukrainische Verfassungsgericht ein Urteil, das Teile der Antikorruptionsreform in der Ukraine außer Kraft setzt. Das Gericht befand zentrale Elemente der ukrainischen Gesetzgebung zur Korruptionsbekämpfung für nicht verfassungskonform, vor allem die strafrechtliche Verfolgbarkeit falscher Angaben durch Staatsbedienstete in den jährlich fälligen Vermögensdeklarationen.

Das Urteil rief heftige Gegenreaktionen beim Präsidenten und der Zivilgesellschaft hervor, die auf eine sich entfaltende Verfassungskrise in der Ukraine hindeuten. Während die krisenhaften Ereignisse sich noch entwirren, ist bereits klar, dass in ihrem Verlauf weder das System zur Bekämpfung der Korruption noch die Integrität des Justizsystems kompromittiert werden dürfen.

\section{Einleitung}

Seit dem gesellschaftlichen Wandel nach dem Euromaidan 2014 fordern die politischen Eliten in der Ukraine die Bekämpfung der Korruption, einem großen Problem im öffentlichen Leben der Ukraine. Diese Bekämpfung erfolgte zweigleisig: Zum einen durch die Schaffung einer institutionellen Infrastruktur, und zum anderen durch einen entsprechenden rechtlichen Rahmen, zu dem unter anderem elektronische Vermögensdeklarationen zur Erfassung illegaler Vermögensanteile durch Staatsbedienstete zählen. Im Rahmen der institutionellen Entwicklung wurden neue Behörden der Exekutive und des Strafverfolgungssystems geschaffen: die Nationale Behörde zur Korruptionsprävention (NAPC), das Nationale Antikorruptionsbüro (NABU) und die Sonderstaatsanwaltschaft für Korruptionsbekämpfung (SAPO). Die Gründung des Hohen Antikorruptionsgerichts (HACC), das für Strafverfahren und Berufungen im Bereich Korruptionsvorwürfe zuständig ist, markierte 2018 den Höhepunkt der Reform des institutionellen Rahmens zur Korruptionsbekämpfung. Außerdem wurde mit der Gründung dieses Gerichts die Aufgabenteilung der Bereiche Ermittlung (NABU, NAPC), Verfolgung (SAPO) und Verurteilung (HACC) von Korruption komplettiert. Was den rechtlichen Rahmen anbelangt, so wurde Ende 2014 von der Werchowna Rada das Gesetz zur Korruptionsprävention verabschiedet, das die europäischen Ambitionen der Ukraine bekräftigte. Mit dem jüngst gefällten Urteil des Verfassungsgerichts wurden zentrale Teile dieser Infrastruktur nun gekippt.

\section{Die umstrittene Entscheidung des Verfassungsgerichts}

Das Urteil geht auf die Klage einer Gruppe von Parlamentsabgeordneten der prorussischen Oppositionsplatt- form Für die Zukunft im August 2020 zurück (welche als Interessenvertretung des Oligarchen Ihor Kolomojskyj in der Rada gilt). Diese Gruppe rief wegen des Vorwurfs der Verfassungswidrigkeit der Antikorruptionsreform von 2014 das Gericht an. Die prorussischen politischen Kräfte versuchen immer wieder, gerichtlich gegen den proeuropäischen Kurs der ukrainischen Regierung vorzugehen und so Errungenschaften des Maidans rückgängig zu machen (beispielsweise ist vor dem Gerichtshof auch noch ein separates Verfahren wegen Verfassungsmäßigkeit des Lustrationsgesetzes von 2014 anhängig).

Die Klage der Abgeordnetengruppe vom August hatte gute Aussichten, durch das Gericht bestätigt zu werden. Tatsächlich veranlasste die Gruppe das Gericht nicht zum ersten Mal, ein Urteil über Antikorruptionsmaßnahmen zu fällen. Bereits im Februar 2019 kassierte das Gericht die strafrechtliche Haftung von Staatsbediensteten im Fall von deren illegaler Bereicherung, wobei es zur Begründung eine ausgesprochen technische Argumentation übernahm: Es entschied, dass die Verpflichtung von Staatsbediensteten, die Rechtmäßigkeit ihres Einkommens nachzuweisen, diese übermäßig belaste und die Unschuldsvermutung ihnen gegenüber außer Kraft setze. Zur Erläuterung erklärte das Gericht, ein fehlender Nachweis über die Rechtmäßigkeit eines Einkommens sei nicht mit der illegalen Erlangung der Vermögenswerte gleichzusetzen und eine Kriminalisierung der entsprechenden Tätigkeiten daher nicht rechtskonform. Die Logik dieser Entscheidung von 2019 ist auch in der neuen Entscheidung des Gerichts erkennbar.

Auch mit ihrem aktuellen Urteil geben die Verfassungsrichter den Klägern recht. Zusammenfassend kann man sagen, dass das Gericht in seiner Entscheidung (http://ccu.gov.ua/sites/default/files/docs/13_r_2020. doc) die Antikorruptionsreform von 2014 im Hinblick 
auf die Unabhängigkeit der Justiz beurteilt. Denn nach Ansicht des Gerichts sieht die Verfassung besondere Garantien für die Judikative vor, die diese gegen Eingriffe und Beeinträchtigungen der Exekutive in hohem Maße schützen.

Das Urteil zählt diese institutionellen Garantien für die richterliche Unabhängigkeit auf und argumentiert, institutionelle Gewaltenteilung bedeute, dass Gerichten und Richtern ein besonders hoher Schutz vor behördlicher Kontrolle durch die Exekutive zukomme. Für den Bereich Korruptionsprävention bedeute dies, dass die Disziplinierung von Richtern und die Überwachung von deren Tätigkeiten durch Organe der Judikative selbst garantiert werden sollten (etwa über eine gerichtliche Selbstkontrolle). In Bezug auf die existierende Antikorruptions-Gesetzgebung stellte das Gericht fest, dass mit der Nationalen Behörde zur Korruptionsprävention ein Regierungsorgan befugt sei, Gerichte und das Verfassungsgericht der Ukraine zu kontrollieren. Im Einzelnen erklärte das Gericht, die Bestimmungen über die Befugnisse der NAPC, Vermögensdeklarationen von Staatsbediensteten einzufordern, aufzubewahren und zu veröffentlichen, gäben der Exekutive ein Mittel zur Kontrolle des Justizapparats an die Hand. Entsprechend hob das Gericht die gesetzlichen Regelungen in Bezug auf die genannten Rechte der Korruptionspräventionsbehörde auf und stellte fest, dass eine andere rechtliche Regelung geschaffen werden müsse, die die besonderen verfassungsrechtlichen Garantien für die Judikative berücksichtigt.

Der umstrittenste Punkt des Urteils betrifft die strafrechtliche Verfolgbarkeit der Nichtabgabe der jährlichen Einkommensdeklarationen durch Staatsbedienstete sowie wissentlich getätigter Falschangaben in diesen Erklärungen - das Gericht schaffte Artikel 366-1 des Strafgesetzbuchs ab, der 2014 mit dem Gesetz zur Korruptionsprävention eingeführt wurde. Die Verfassungsrichter argumentieren in diesem Zusammenhang, dass es zur Rechtfertigung einer strafrechtlichen Verfolgung der in Frage stehenden Vorgänge keine ausreichenden Beweise gäbe bzw. dass - anders ausgedrückt - die entsprechenden Strafen in keinem angemessenen Verhältnis zu dem gesellschaftlichen Schaden stünden, den fehlende oder falsche Angaben in Vermögensdeklarationen verursachten. Nach Meinung des Gerichts sollte die strafrechtliche zu einer verwaltungsrechtlichen Haftung herabgestuft werden. Insgesamt stellt das Urteil des Gerichts eine Aushöhlung der Antikorruptionsreform dar, indem es die wichtigsten Regelungen des Gesetzes von 2014 antastet. Bedeutsam ist das Urteil außerdem, weil die nun aufgehobene Gesetzgebung eine zentrale Bedingung für die Visa-Liberalisierung der EU und die Gewährung finanzieller Unterstützung für die Ukraine durch internationale Partner war.

\section{Der Aufschrei in der Ukraine und internationale Reaktionen}

In der Ukraine löste die Entscheidung des Gerichts beim Präsidenten und der Zivilgesellschaft eine erbitterte und heftige Gegenwehr aus, die zeigt, wie wenig glaubwürdig das Verfassungsgericht bei Öffentlichkeit und Politik ist. Präsident Selenskyj bezog als Antwort auf die Ereignisse einen kompromisslos konfrontativen Standpunkt gegenüber dem Gericht und drohte den Verfassungsrichtern, sie aus ihren Ämtern zu entheben. Und es blieb nicht nur bei Worten des Präsidenten: Er legte dem Parlament einen Gesetzentwurf vor, der diesem das Recht geben soll, das umstrittene Urteil zu annullieren und alle Verfassungsrichter aus ihren momentanen Ämtern zu entfernen. Im Augenblick scheint dieser Ruf des Präsidenten zu verhallen, ohne dass der zuständige Parlamentsausschuss reagiert. Zusätzlich forderte Selenskyj die Richter öffentlich auf, ihre Ämter freiwillig niederzulegen. Hierzu ist festzuhalten, dass dem Gericht laut Verfassung ein besonderer Status zukommt - weder Präsident noch Parlament können Verfassungsrichter entlassen. Zudem enthält das Gesetz über das Verfassungsgericht von 2017 eine Liste, die exklusiv festlegt, wann das Mandat von Verfassungsrichtern endet (etwa, wenn diese 70 Jahre alt werden oder eine ausländische Staatsbürgerschaft annehmen).

Interessant zu sehen ist in diesem Zusammenhang, dass zivilgesellschaftliche Akteure im Bereich der Korruptionsbekämpfung den Plan des Präsidenten nicht als einen ungeheuerlichen Fall von Einmischung in die Judikative bewertet haben oder zugunsten der Unabhängigkeit des Gerichts intervenierten. Im Gegenteil - viele äußerten heftige Kritik an den Verfassungsrichtern, die die korrupten politischen Eliten decken würden. Dieser Vorwurf richtete sich speziell an die Richter, die in der Janukowytsch-Ära ernannt wurden, vor allem an den derzeitigen Gerichtspräsidenten Oleksandr Tupizkyj, der seit 2013 Verfassungsrichter ist.

Hinzu kommt, dass Meinungsführer wie Witalij Schabunin von der NGO "Zentrum zur Korruptionsbekämpfung « und Serhij Leschtschenko, ehemaliger Parlamentsabgeordneter und früherer Investigativjournalist - beide sind für ihr öffentliches Engagement im Bereich Antikorruption sehr bekannt -, in den Medien kompromisslos Stellung gegen Gericht und Verfassungsrichter bezogen haben. Auch Expertencommunitys wie das »Reanimation Package of Reforms « und die Stiftung "De Jure« verurteilten das Gericht wegen Sabotage der Antikorruptionsreform. In ihren Erklärungen konstatierten die Experten eine mangelnde juristische Begründung durch die Verfassungsrichter und bezeichneten ihr Urteil als unbegründet. Beide Expertencommunitys forderten die Richter, die dem Urteil zugestimmt haben, zur freiwilligen Aufgabe ihrer Ämter auf. 
Um diese Reaktion der Zivilgesellschaft zu verstehen, hilft es, einen Blick auf den politischen Kontext der Justiz werfen. Die ukrainischen Gerichte galten in der Öffentlichkeit lange Zeit als wenig vertrauenswürdig. Das gilt für die Jahre vor wie nach dem Euromaidan, wobei Umfragen eine kleine Veränderung der öffentlichen Einstellung gegenüber Richtern und Gerichten zeigen. In besonderem Maße gilt dies für das Verfassungsgericht, denn diese Institution hat schon in der Janukowytsch-Ära starken Gegenwind für ihre Urteile erfahren und war durch die Entmachtung einiger amtierender Richter per Parlamentsentscheidung in der Folge der revolutionären Umwälzungen in der Ukraine 2014 eine Zeitlang handlungsunfähig.

Nach dem Euromaidan wurden in der Ukraine zahlreiche Gesetze eingeführt, um die institutionelle Verfasstheit der ukrainischen Justiz zu reformieren. Insbesondere wurde unter Präsident Petro Poroschenko eine große Verfassungsreform verabschiedet. Zwischen 2016 und 2018 folgten neue Gesetze zum Gerichtssystem, der Besetzung von Richterstellen und dem Verfassungsgericht. Ziel dieser Justizreform war es, den Prozess der Besetzung von Richterstellen zu entpolitisieren, die professionellen Standards innerhalb des Gerichtswesens anzuheben und die Unabhängigkeit der Judikative zu erhöhen. Fraglich bleibt angesichts der Situation rund um die ukrainischen Gerichte, warum die weitreichenden institutionellen Veränderungen in den Jahren nach dem Euromaidan keine größere öffentliche Akzeptanz der Justiz als glaubwürdige Institution in der Ukraine bewirkt haben.

Selbst dass 2017 der Rahmen für die verfassungsmäßige Gerichtsbarkeit reformiert und 2016 und 2018 neue Richter ernannt wurden, verbesserte in der breiten Öffentlichkeit weder die öffentliche Wahrnehmung des Gerichts noch das Bild von dessen professioneller und ethischer Integrität. Vor diesem Hintergrund ist es zu verstehen, dass Akteure der Zivilgesellschaft, von denen man annehmen würde, dass sie das Prinzip der Rechtsstaatlichkeit und die Judikative gegen deren Beeinflussung durch politische Entscheidungsträger verteidigen würden, ihre Stimme in diesem speziellen Fall gegen das Gericht erheben. Auch angesichts der Aussicht, dass die Ukraine aufgrund des Gerichtsurteils sogar die Visumfreiheit mit der EU aufs Spiel setzt, sorgen sich die zivilgesellschaftlichen Akteure stärker um die Integrität des Antikorruptionssystems als um eine Verletzung rechtsstaatlicher Prinzipien.

Mit Blick auf die internationale Gemeinschaft ist die Reaktion der Delegation der Europäischen Union für die Ukraine zu nennen, die die ukrainische Regierung in einer Erklärung aufgefordert hat, die vom Gericht gekippte Gesetzgebung so schnell wie möglich wiederherzustellen. Außerdem wies die Delegation darauf hin, dass Antikorruptionsmaßnahmen ein zentraler Prüfstein für den Prozess der Visaliberalisierung und für die Gewährung von Makrofinanzhilfen an die Ukraine sind. Außerdem ließ Mykola Tochyzkyj, der ukrainische Botschafter bei der EU, die ukrainische Regierung wissen, dass die Abschaffung der Antikorruptions-Gesetzgebung die Visaliberalisierung mit der EU gefährde und die Europäische Kommission die Visafreiheit zwischen EU und Ukraine vorläufig aussetzen könne. Das heißt jedoch nicht, dass die Kommission solche Schritte auch tatsächlich unternimmt. Im Gegenteil - die Delegation der Europäischen Union in der Ukraine scheint Nuancen der momentanen Situation wahrzunehmen und außer der Warnung eines ukrainischen Diplomaten gibt es keinen Hinweis darauf, dass Brüssel die Visafreiheit mit der Ukraine aussetzen könnte.

Darüber hinaus meldeten sich die Präsidenten der Venedig-Kommission und der Staatengruppe gegen Korruption des Europarates (GRECO) in einem gemeinsamen Brief an das Parlament der Ukraine zu Wort. In diesem fordern sie das Parlament dringend auf, einen Weg aus der Krise zu finden, der ohne drastische Maßnahmen zur Kompromittierung des Verfassungsgerichts auskommt, und weisen darauf hin, dass die Beendigung von Amtszeiten von Verfassungsrichtern einen ungeheuerlichen Eingriff in die richterliche Unabhängigkeit darstellen würde, der langanhaltende negative Folgen für die Rechtsstaatlichkeit in der Ukraine nach sich zöge. Eigenartigerweise ist sich die internationale Expertengemeinschaft des Gedankens an die Rechtsstaatlichkeit also stärker bewusst als die zivilgesellschaftlichen Akteure in der Ukraine.

\section{Kein Ausweg?}

Angesichts der sich entfaltenden Verfassungskrise ist klar, dass nur die Werchowna Rada eine legitime Entscheidung herbeiführen kann. Um vor diesem Hintergrund einen Weg aus der Krise zu finden, haben der Präsident und die Abgeordneten einige wenige drastische Optionen sowie eine mildere Option vorgestellt. Der vom Präsidenten geforderten Amtsenthebung der Verfassungsrichter wird das Parlament angesichts der weiteren Kompromittierung der Judikative, die ein derartiges Gesetz bedeuten würde, wohl nicht zustimmen - auch wenn eine solche Entscheidung Anklang bei jenen Teilen der Zivilgesellschaft finden könnte, die von der grundlegenden Kehrtwende in der Korruptionsbekämpfung enttäuscht sind.

Das gilt auch für Ideen, die Arbeit des Gerichts durch eine Anhebung der zur Beschlussfähigkeit nötigen Anzahl von Richtern zu blockieren. Einem Aufruf des Präsidenten folgend legte eine Gruppe von Abgeordneten einen Entwurf für ein solches Gesetz vor, das die Anzahl der für die Beschlussfähigkeit des Gerichts 
erforderlichen Richter von 12 auf 17 erhöhen könnte. In der momentanen Situation, in der 15 von 18 gesetzlich vorgesehenen Richterstellen besetzt sind, würde so jegliche Entscheidung durch das Gericht effektiv verhindert. Dies wäre allerdings eine kurzfristige und erneut sehr umstrittene Lösung.

Zum Zeitpunkt der Einreichung dieses Textes verweigerten vier Verfassungsrichter - Serhij Holowatyj (ernannt 2018), Oleh Perwomajskyj (2018), Wiktor Kolesnik (2016) und Wassyl Lemak (2018) - ihre Teilnahme an Gerichtsverhandlungen und verhinderten so das Zustandekommen verfassungsrechtlicher Entscheidungsfindungsprozesse. Sie alle wurden während Poroschenkos Amtszeit ernannt, sie alle stimmten gegen die Entscheidung über die Antikorruptionsreform und begründeten ihre Standpunkte voneinander abweichend. Die Weigerung der Richter, an der Arbeit teilzunehmen, führt dazu, dass die Große Kammer des Gerichts momentan nicht tagen und über die Verfassungsmäßigkeit normaler Gesetze entscheiden kann. Ihre Abwesenheit verhinderte die befürchtete Umkehr weiterer Reformen, die bereits auf der Tagesordnung des Gerichts standen - unter anderem das Gesetz zur Landreform und das Sprachengesetz.

Zur Beruhigung und als Ausweg aus der Krise stellte Parlamentssprecher Dmytro Rasumkow dem Parlament eine dritte Option vor. Das sogenannte RasumkowProjekt sieht eine Verfügung vor, die die wichtigsten Bestimmungen der Antikorruptions-Gesetzgebung mit Wirkung ab dem 27. Oktober (dem Tag, an dem das Gericht seine Entscheidung verkündete) wieder in Kraft setzt. Die Rechtsabteilung der Rada kritisierte diese Lösung jedoch, da Entscheidungen des Verfassungs- gerichts laut Verfassung zwingend umgesetzt werden müssen. Insofern kann die Rada abgeschaffte Bestimmungen nicht einfach wieder in Kraft setzen, als habe es kein Gerichtsurteil über sie gegeben. Das bedeutet, dass die Rada die Gerichtsentscheidung substanziell durcharbeiten muss, um deren Logik und die Begründungen der Richter zu erfassen. Außerdem könnte im Fall seiner Verabschiedung auch das Rasumkow-Gesetz für nicht verfassungskonform erklärt werden.

Angesichts des Umstands, dass das Verfassungsgericht momentan wohl weniger Rückhalt in der Bevölkerung hat als jemals zuvor in der jüngeren Geschichte, sollte eine ausgewogene Entscheidung gefunden werden. Einerseits ist die allgemeine Stimmung in der Öffentlichkeit, der zufolge die Antikorruptionsreform erhalten bleiben sollte, verständlich. Die Bekämpfung der Korruption ist ein zentrales Versprechen der Post-MaidanEliten an die ukrainische Gesellschaft. Nichtsdestotrotz fehlt in der öffentlichen Diskussion der Ereignisse, die das Urteil in der Ukraine ausgelöst hat, häufig der Blick darauf, dass auch die Rechtsstaatlichkeit in dieser Krise auf dem Spiel steht. Die Integrität der Judikative darf durch außerordentliche und drastische Parlamentsentscheidungen keinesfalls weiter kompromittiert werden. Stattdessen ist es wohl vielmehr tatsächlich so, dass die Rechtsexperten der Werchowna Rada die tragfähigste und eine zugleich rechtskonforme Lösung vorgeschlagen haben - das Parlament sollte sich durch die Kritik des Gerichts hindurcharbeiten und diese erfassen, um anschließend einen neuen Gesetzentwurf zur Bekämpfung der Korruption in der Ukraine vorzulegen.

Übersetzung aus dem Englischen von Sophie Hellgardt

\section{Über den Autor}

Andrii Nekoliak ist Doktorand am Johan Skytte Institute of Political Studies an der Universität von Tartu in Estland. Er verfügt über Abschlüsse in Politikwissenschaften und Jura und hat ein Kapitel zur Sprachpolitik im ukrainischen Verfassungsgericht für einen bei Palgrave MacMillan erschienenen Band verfasst.

\section{Lesetipps}

- Lovin, Anton und Ruslana Vovk. 2018. "Taking Stock: First Steps of the Renewed Constitutional Court of Ukraine." Democracy Reporting International. https://democracy-reporting.org/ru/ taking-stock-first-steps-of-the-renewed-constitutional-court-of-ukraine/

- Nekoliak, Andrii und Vello Pettai. 2020. „Navigating Ethnopolitical Disputes: Ukraine's Constitutional Court in the Tug-of-War over Language." In Decentralization, Regional Diversity, and Conflict: The Case of Ukraine, herausgegeben von Maryna Rabinovych und Hanna Shelest. Palgrave Macmillan, S. 49-79

- Popova, Maria und Daniel J. Beers. 2020. "No Revolution of Dignity for Ukraine’s Judges: Judicial Reform after the Euromaidan." Demokratizatsiya: The Journal of Post-Soviet Democratization 28: 1 (Winter 2020), S. 113-142

- Popova, Maria; Zhernakov, Mykhailo. 2020. Das Trugbild vom Durchbruch zum Rechtsstaat: Justizreform nach der Revolution der Würde. Ukraine-Analysen 238, https://doi.org/10.31205/UA.238.02. 


\section{Chronologie der Verfassungskrise, 27. Oktober - 06. November 2020}

\begin{tabular}{|c|c|}
\hline 27.10.2020 & $\begin{array}{l}\text { Das Verfassungsgericht der Ukraine erklärt in einer geschlossenen Sitzung die Veröffentlichung der elektroni- } \\
\text { schen Vermögensdeklarationen von Top-Beamten und deren Kontrolle durch die zuständige Nationale Agentur } \\
\text { zur Prävention von Korruption (NAPC) als verfassungswidrig. Damit müssen das NAPC und das für Ermitt- } \\
\text { lungen zuständige Nationale Antikorruptionsbüro (NABU) mehr als hundert Verfahren, auch gegen hoch- } \\
\text { rangige mutmaßlich korrupte Richter, einstellen und die elektronische Datenbank mit den Vermögensdekla- } \\
\text { rationen vom Netz nehmen. Weiterhin kippt das Gericht Artikel 366-1 des Strafgesetzbuchs, der Falschanga- } \\
\text { ben in den Vermögensangaben unter Strafe stellt. Die beiden Entscheidungen, die auf Initiative einer Gruppe } \\
\text { von Abgeordneten mit Verbindungen zu Oligarchen angestoßen wurden, stoßen auf große Kritik aus Politik, } \\
\text { Gesellschaft und dem Ausland und stürzen das Land in eine Verfassungskrise. }\end{array}$ \\
\hline 28.10 .2020 & $\begin{array}{l}\text { Der Präsident des Verfassungsgerichts der Ukraine, Oleksandr Tupizkyj, soll } 2018 \text { illegal auf der von Russland } \\
\text { annektierten Krim ein Grundstück erworben und anschließend nicht deklariert haben, berichtet das bekannte } \\
\text { Investigativ-Programm »Schemy«. Damit läge ein klarer Interessenkonflikt mit den am Vortrag gefällten und } \\
\text { umstrittenen Entscheidungen des Verfassungsgerichts vor, die die juristische Haftung in solchen Fällen abschafft. }\end{array}$ \\
\hline 29.10 .2020 & $\begin{array}{l}\text { Präsident Wolodymyr Selenskyj ruft wegen der Entscheidung des Verfassungsgerichts, Teile der Antikorrupti- } \\
\text { onsinfrastruktur als verfassungswidrig zu erklären, den Sicherheits- und Verteidigungsrat zu einer Dringlich- } \\
\text { keitssitzung zusammen, und stuft die umstrittene Entscheidung als eine Bedrohung und Herausforderung für } \\
\text { die nationale Sicherheit ein. }\end{array}$ \\
\hline 29.10 .2020 & $\begin{array}{l}\text { Das Ministerkabinett beschließt auf einer außerordentlichen Sitzung auf Empfehlung des Nationalen Sicher- } \\
\text { heits- und Verteidigungsrates eine Eilverfügung. Darin wird die Nationale Agentur zur Prävention von Korrup- } \\
\text { tion (NAPC) dazu verpflichtet, die elektronischen Vermögensdeklarationen unverzüglich wieder offen zugäng- } \\
\text { lich zu machen. Am Abend des 29. Oktober kommt die NAPC der Verfügung nach und stellt die elektroni- } \\
\text { schen Vermögensdeklarationen wieder offen ins Netz. }\end{array}$ \\
\hline 29.10 & $\begin{array}{l}\text { Das Staatliche Ermittlungsbüro (DBR) gibt bekannt, gegen den Präsidenten des Verfassungsgerichts der } \\
\text { Ukraine, Oleksandr Tupizkyj, wegen der Zugehörigkeit zu einer »kriminelle Organisation« zu ermitteln. Für } \\
\text { den 2. November wird Tupizkyj zu einem Gespräch beim DBR vorgeladen. }\end{array}$ \\
\hline 30.10 .2020 & $\begin{array}{l}\text { Präsident Wolodymyr Selenskyj reicht im Parlament einen Gesetzesentwurf "Zur Wiederherstellung des Ver- } \\
\text { trauens der Öffentlichkeit in Verfassungsprozesse« ein. Der Entwurf sieht vor, die umstrittenen Urteile des } \\
\text { Verfassungsgerichts als "nicht rechtskräftig» aufzuheben und alle Richter des Verfassungsgerichts zu entlas- } \\
\text { sen. Damit soll der Weg frei gemacht werden für eine neue Zusammensetzung der Verfassungsrichter, die zum } \\
\text { Teil noch unter Ex-Präsident Wiktor Janukowytsch ernannt wurden. Selenskyjs Vorschlag ist umstritten, da } \\
\text { weder der Präsident noch das Parlament formal berechtigt sind, Verfassungsrichter zu entlassen. Diese kön- } \\
\text { nen nur selbst zurücktreten. }\end{array}$ \\
\hline 30.10 .2020 & $\begin{array}{l}\text { Der Präsident des Verfassungsgerichts der Ukraine, Oleksandr Tupizkyj, kritisiert den Gesetzesentwurf von } \\
\text { Präsident Wolodymyr Selenskyj und bezeichnet diesen als »Staatsstreich«. }\end{array}$ \\
\hline 30.10 .2020 & $\begin{array}{l}\text { Vor dem Gebäude des Verfassungsgerichts kommt es zu Protesten. Die Organisatoren, darunter mehrere Anti- } \\
\text { korruptions-NGOs, fordern den sofortigen Rücktritt der Verfassungsrichter. Unter anderem werden brennende } \\
\text { Reifen auf das Gebäude geworfen. }\end{array}$ \\
\hline 31.10 .2020 & $\begin{array}{l}\text { Die Venedig-Kommission und GRECO (Staatengruppe gegen Korruption des Europarates) mahnen die Wer- } \\
\text { chowna Rada, dass die Verabschiedung des Gesetzesentwurfs von Präsident Wolodymyr Selenskyj, der die } \\
\text { Entlassung der Verfassungsrichter vorsieht, gegen die Verfassung und internationales Recht verstoße und die } \\
\text { Gewaltenteilung untergrabe. Sie fordern das Parlament dazu auf, einen anderen Weg zur Beendigung der Ver- } \\
\text { fassungskrise zu finden und bieten Parlamentssprecher Dmytro Rasumkow Hilfe bei der Ausarbeitung einer } \\
\text { verfassungskonformen Lösung an. }\end{array}$ \\
\hline 02.11 .2020 & $\begin{array}{l}\text { Präsident Wolodymyr Selenskyj fordert bei einem Fernsehauftritt das Parlament auf, seinem Gesetzesentwurf } \\
\text { zur Entlassung der Verfassungsrichter zuzustimmen und schließt nicht aus, andernfalls das Parlament aufzulö- } \\
\text { sen, sollte die Verfassungskrise nicht anders gelöst werden können. So soll das Land vor dem "politischen Kol- } \\
\text { laps« bewahrt werden, so Selenskyj. Er wirft indirekt Oligarchen und pro-russischen Politikern vor, die Ent- } \\
\text { scheidungen des Verfassungsgerichts beeinflusst zu haben, um die Beziehungen der Ukraine zu ihren westli- } \\
\text { chen Partnern zu untergraben. }\end{array}$ \\
\hline 03.11 .2020 & $\begin{array}{l}\text { Der Sprecher der EU für Außen- und Sicherheitspolitik, Peter Stano, kritisiert die umstrittene Entscheidung } \\
\text { des Verfassungsgerichts vom 27. Oktober. Die Ukraine habe sich im Rahmen des Assoziierungsabkommens, } \\
\text { dem makrofinanziellen EU-Hilfsprogramm und der Visaliberalisierung der Korruptionsbekämpfung verpflich- } \\
\text { tet, und die Entscheidung gefährde diese Verpflichtungen. }\end{array}$ \\
\hline
\end{tabular}




\begin{tabular}{|l|l|}
\hline 03.11.2020 & $\begin{array}{l}\text { Das Oberste Antikorruptionsgericht muss aufgrund der gekippten Entscheidung des Verfassungsgerichts zu } \\
\text { Falschangaben in elektronischen Vermögensdeklarationen den ersten Fall schließen: Der ehemalige Vorsitzende } \\
\text { der Region Saporischschja, Kostjantyn Bryl, stand vor Gericht, da er fünf Kraftfahrzeuge, darunter ein Auto } \\
\text { der Marke Range Rover sowie fünf der Marke Mercedes Benz, nicht deklariert hatte. }\end{array}$ \\
\hline 03.11 .2020 & $\begin{array}{l}\text { Laut einer repräsentativen Umfrage des Rating-Instituts misstrauen 60 Prozent der Ukrainer dem Verfassungs- } \\
\text { gericht, während 19 Prozent dem Gericht vertrauen. Die durch die Entscheidung des Verfassungsgerichts mög- } \\
\text { liche Aufhebung der Visaliberalisierung würden 55 Prozent als negativ betrachten; 11 Prozent würden sie hin- } \\
\text { gegen begrüßen. }\end{array}$ \\
\hline 04.11 .2020 & $\begin{array}{l}\text { Finanzminister Serhij Martschenko warnt, dass die Entscheidung des Verfassungsgerichts vom 27. Oktober } \\
\text { die Makrofinanzhilfen und die Kredite des IWF, der EU und der Weltbank gefährden, die der Ukraine für } \\
\text { 2021 bereits zugesichert wurden. }\end{array}$ \\
\hline 05.11 .2020 & $\begin{array}{l}\text { Das Oberste Antikorruptionsgericht schließt nach der Entscheidung des Verfassungsgerichts vom 27. Oktober } \\
\text { den Fall gegen den Bürgermeister von Odessa, Hennadij Truchanow. Truchanow wird vorgeworfen, Vermö- } \\
\text { genswerte in Höhe von 16 Mio. Hrywnja (ca. 470.000 Euro) nicht deklariert zu haben. }\end{array}$ \\
\hline $\begin{array}{l}\text { Das Verfassungsgericht der Ukraine verschiebt eine für heute geplante Abstimmung über die Landreform, } \\
\text { da das nötige Quorum - mindestens 12 der 18 Verfassungsrichter müssen anwesend sein - nicht erreicht sei. } \\
\text { Medienberichten ukrainischer Zeitungen zufolge wollen einige Verfassungsrichter mit ihrem Fernbleiben ver- } \\
\text { hindern, dass weitere Reformen vom Verfassungsgericht rückgängig gemacht werden. Da aktuell drei Posten } \\
\text { vakant sind, müssen vier Richter fernbleiben, damit die Beschlussfähigkeit nicht erreicht wird. Genau vier } \\
\text { Richter erklären, dass sie temporär nicht an den Sitzungen teilnehmen werden, womit die Arbeit des Verfas- } \\
\text { sungsgerichts vorerst blockiert ist. }\end{array}$ \\
\hline
\end{tabular}

Quelle: Chronik der Ukraine-Analysen vom 27.10 - 06.11.2020, https://www.laender-analysen.de/ukraine-analysen/chronik.

\section{Die Richter des Verfassungsgerichts und ihr Abstimmungsverhalten am 27. Oktober 2020}

Tabelle 1: Die Richter des Verfassungsgerichts und ihr Abstimmungsverhalten am 27. Oktober 2020

\begin{tabular}{|c|c|c|c|c|}
\hline Name & Ernannt wann & Ernannt von & $\begin{array}{c}\text { Stimmte für } \\
\text { Verfassungswidrigkeit der } \\
\text { Antikorruptionsgesetze }\end{array}$ & $\begin{array}{c}\text { Stimmte gegen } \\
\text { Verfassungswidrigkeit der } \\
\text { Antikorruptionsgesetze }\end{array}$ \\
\hline Filjuk, P. T. & 2019 & Richterrat & $x$ & \\
\hline Holowatyj, S. P. & 2018 & Poroschenko & & $x$ \\
\hline Horodowenko, W. W. & 2017 & Richterrat & $x$ & \\
\hline Jurowska, H. W. & 2019 & Richterrat & $x$ & \\
\hline Kasminin, O. W. & 2013 & Janukowytsch & $x$ & \\
\hline Kolisnyk, W. P. & 2016 & Poroschenko & & $x$ \\
\hline Krywenko, W. W. & 2016 & Richterrat & $x$ & \\
\hline Lemak, W. W. & 2018 & Poroschenko & & $x$ \\
\hline Lytwynow, O. M. & 2013 & Richterrat & $x$ & \\
\hline Mojsyk, W. R. & 2016 & Poroschenko & $x$ & \\
\hline Perwomajskyj, O. O. & 2018 & Parlament & & $x$ \\
\hline Sas, S. W. & 2014 & Parlament & $x$ & \\
\hline Sawhorodnja, I. M. & 2018 & Parlament & $x$ & \\
\hline Slidenko, I. D. & 2014 & Parlament & $x$ & \\
\hline Tupizkyj, O. M. (Präsident) & 2013 & Janukowytsch & $x$ & \\
\hline
\end{tabular}




\section{Vertrauen in die Justiz}

Grafik 1: $\quad$ Vertrauen in Institutionen (in \%)

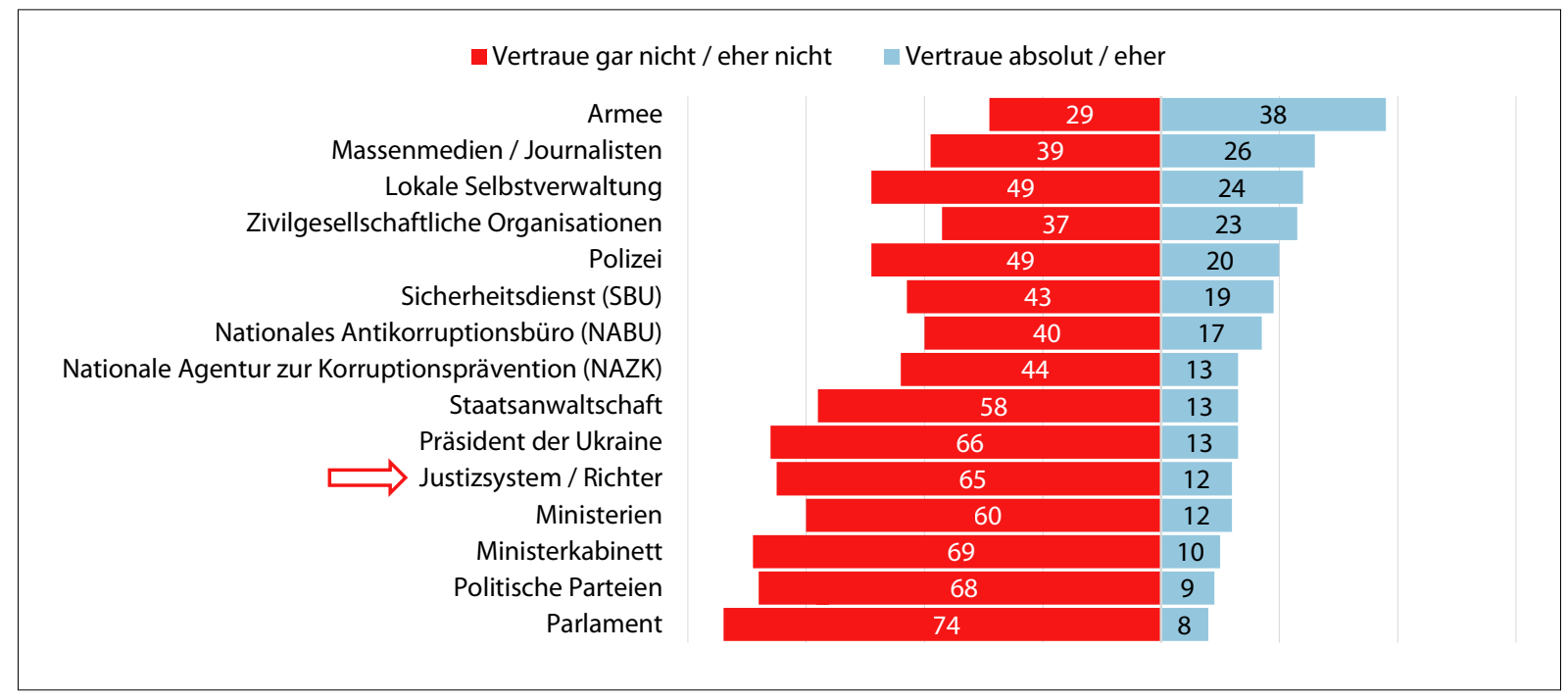

Quelle: Results of USAID New Justice Program 2017 National Surveys of the Public, Judges and Justice Sector Professionals regarding Judicial Reform, Public Trust in the Judiciary, Judicial Independence and Accountability, and Perception of Corruption, 2017, https://newjustice.org.ua/wp-content/uploads/2018/09/NJ_2017_ Survey_Results_Oct2017_ROL_ENG.pdf.

Grafik 2: $\quad$ Vertrauen Sie dem Verfassungsgericht... (in \%)

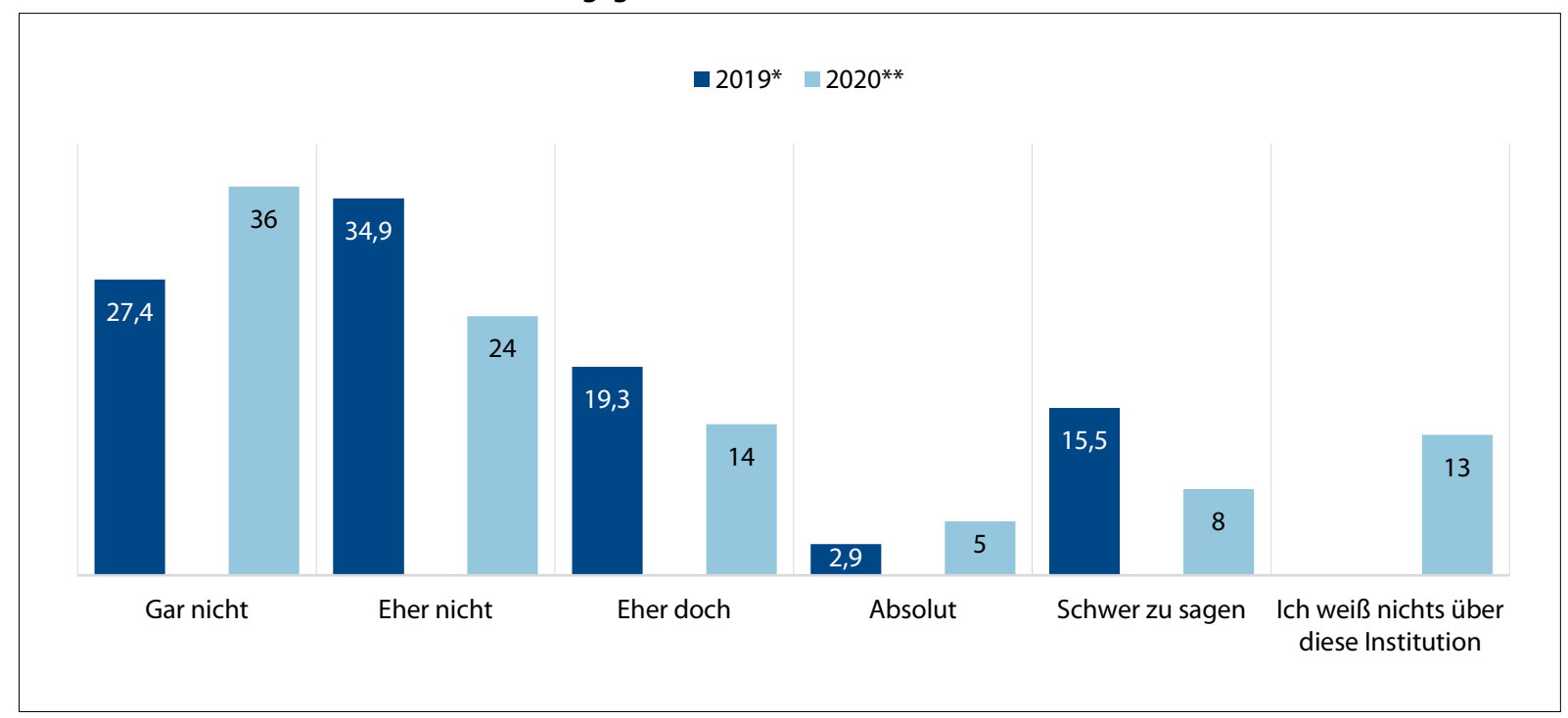

Quellen: * Justizreform in den Augen der_Bürger [Sudowa reforma otschyma hromadjan], 12.07.2019, https://dif.org.ua/en/article/sudova-reforma\#_ftn1 (die_Antwortmöglichkeit Ich weiß nichts über diese Institution gab es in dieser Umfragenicht); ${ }^{*}$ Repräsentative Umfrage des Rating-Instituts, 04.11.2020, http://ratinggroup. ua/research/ukraine/ocenki_ukraincev_poslednih_rezonansnyh_sobytiy.html. 
Grafik 3: $\quad$ Vertrauen in die Justiz (in \%)

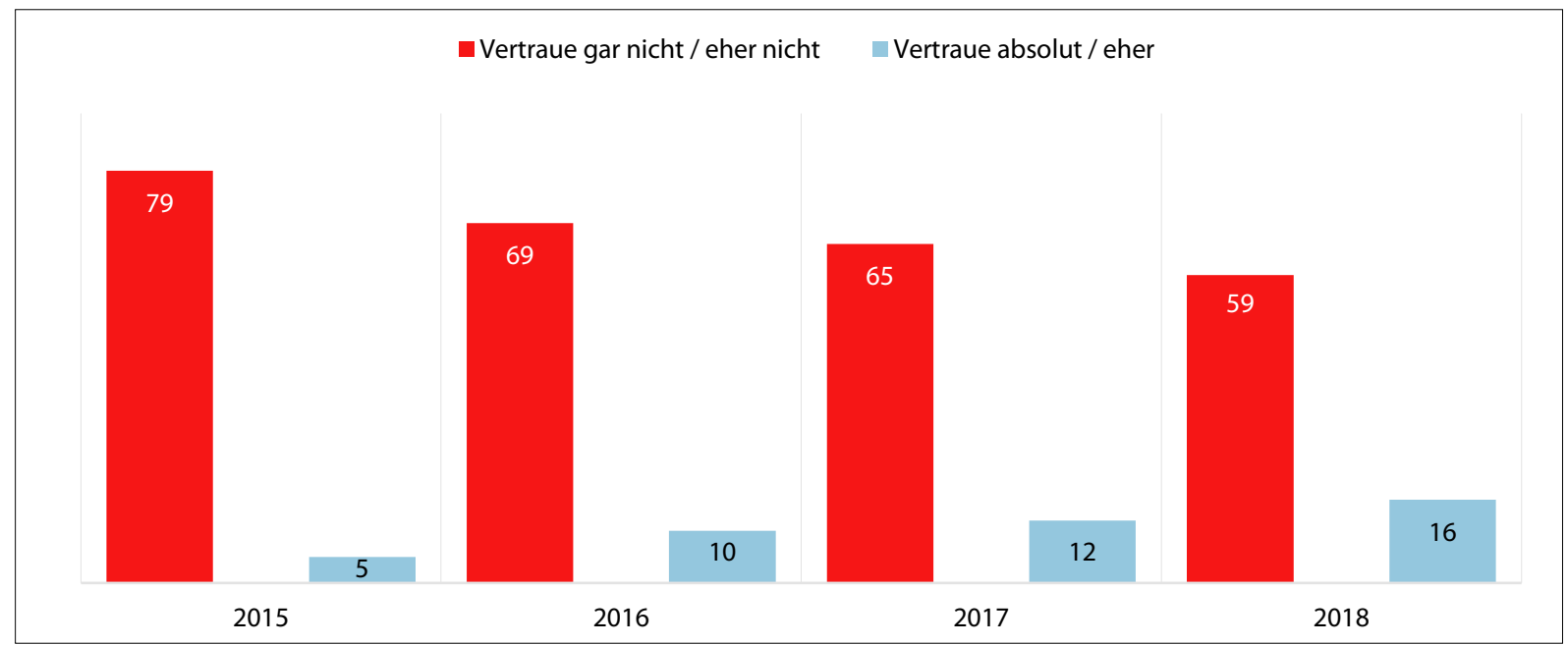

Quelle: Result of the Second National Public Survey Regarding Trust in the Judiciary Judicial Reform and Perception of Corruption, 2018, https://newjustice.org. ua/wp-content/uploads/2018/11/1_NJ_October_2018_SurveyPublic_Results_ENG.pdf.

Grafik 4: Haben Sie von der Entscheidung des Verfassungsgerichts gehört, mit der die E-Deklarationen und der Zugang zu Informationen über Vermögenswerte und Einkommen hochrangiger Beamter abgeschafft wurden? (in \%)

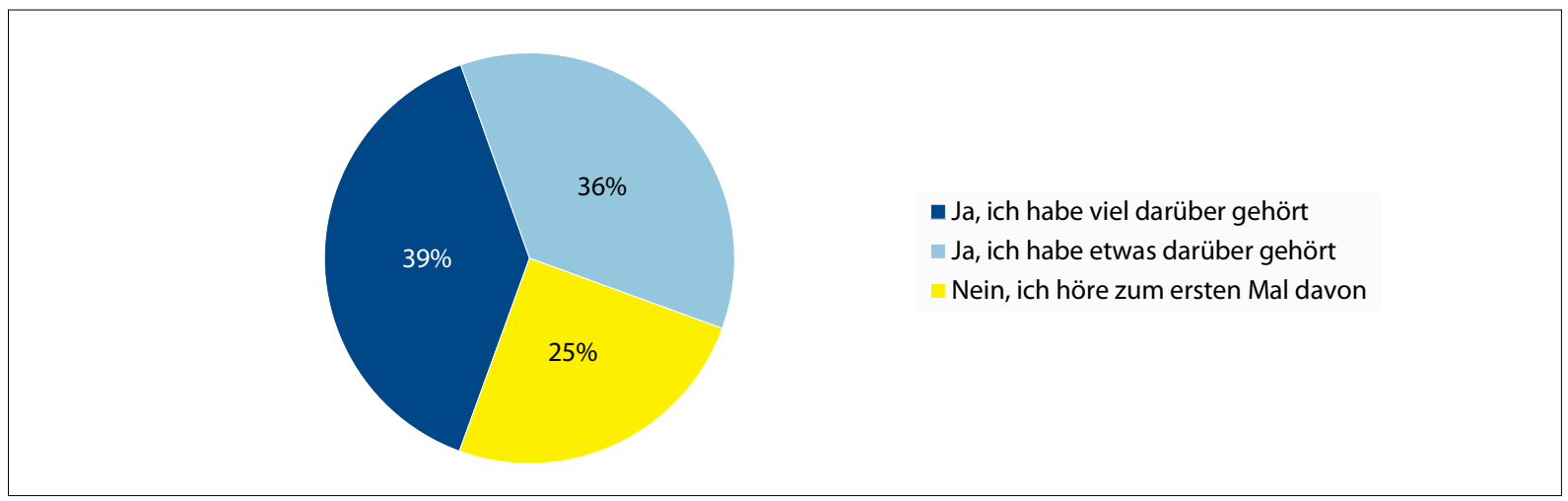

Quelle:Repräsentative Umfrage des Rating-Instituts, 04.11.2020, http://ratinggroup.ua/research/ukraine/ocenki_ukraincev_poslednih_rezonansnyh_sobytiy. html.

\section{Grafik 5: Wie stehen Sie zu dieser Entscheidung des Verfassungsgerichts? (in \%)}

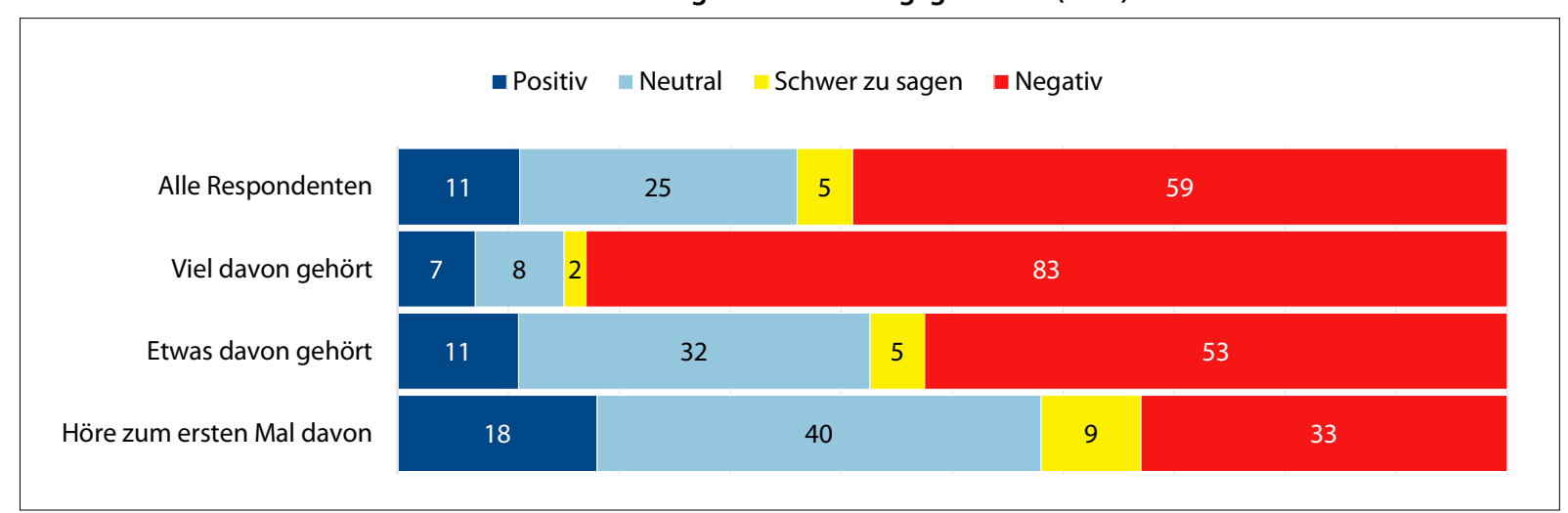

Quelle:Repräsentative Umfragedes Rating-Instituts, 04.11.2020,http://ratinggroup.ua/research/ukraine/ocenki_ukraincev_poslednih_rezonansnyh_sobytiy. html. 


\section{Erklärung zivilgesellschaftlicher Organisationen zur Entscheidung des Verfassungsgerichts}

Erklärung von 43 zivilgesellschaftlichen Organisationen, die nach der umstrittenen Entscheidung des Verfassungsgerichts der Ukraine, einen wichtigen Bestandteil der Antikorruptionsinfrastruktur für verfassungswidrig zu erklären, den freiwilligen Rücktritt der Richter fordern, um die Verfassungskrise zu beenden.

\section{Constitutional Court Judges Must Resign}

We call for the immediate voluntary resignation of all judges of the Constitutional Court of Ukraine (CCU) who supported the dismantling of the anti-corruption reform in Ukraine.

They are: Oleksandr Mykolaiovych Tupytskyi, Viktor Valentynovych Horodovenko, Iryna Mykolaivna Zavhorodnia, Oleksandr Volodymyrovych Kasminin, Viktor Vasylovych Kryvenko, Oleksandr Mykolaiovych Lytvynov, Volodymyr Romanovych Moisyk, Serhii Volodymyrovych Sas, Ihor Dmytrovych Slidenko (filed a letter of resignation), Petro Todosiovych Filiuk, Halyna Valentynivna Yurovska.

Corruption is recognized as one of the threats to national security. Legislative norms which were declared unconstitutional by the decision of the CCU formed the basis of the anti-corruption system. We are outraged by this decision, as it significantly harms the country's reputation and turns the vector of its development around. With their decision, the judges infringed on Ukraine's strategic course towards the EU and NATO, which is set forth in the Constitution of Ukraine and which we achieved after the Revolution of Dignity.

Making this heinous decision, the CCU judges exceeded their powers and issued the decision in the situation of a conflict of interest. The consequences of this decision are irreversible, with over 100 cases considered by the High Anti-Corruption Court and investigated by the National Anti-Corruption Bureau closed. The perpetrators will not be punished. By violating its international commitments under agreements with the IMF, the EU and other international partners, Ukraine risks losing the external financial support that it desperately needs during the ongoing crisis.

In addition to the damage already caused by the judges, now, laws on the language, on the land reform, on the High Anti-Corruption Court and on the Deposit Guarantee Fund are similarly under threat. Petitions about them are already on the CCU agenda, and decisions may be issued very soon.

We are tired of dishonest judges and illegal decisions that are not based on the rule of law and justice. That is why we call on the judges of the Constitutional Court to resign voluntarily.

$\begin{array}{ll}\text { Transparency International Ukraine } & \text { Ukrainian School of Political Studies } \\ \text { Automaidan } & \text { CSO Agency of Efficient Solutions } \\ \text { Anti-Corruption Headquarters } & \text { Independent Defense Anti-Corruption } \\ \text { Center for Public Monitoring and } & \text { Committee (NAKO) } \\ \text { Research } & \text { Institute of Analysis and Advocacy } \\ \text { DEJURE Foundation } & \text { CSO Association of Political Studies } \\ \text { Anti-Corruption Research and Educa- } & \text { Exampei Center for Ukrainian Politics } \\ \text { tion Centre (ACREC) } & \text { CSO Analytical Advocacy Center } \\ \text { CSO Public Television of Svitlovodsk } & \text { Lviv Regulatory Hub } \\ \text { CSO Kremenchuk Anti-Corruption } & \text { Institute of Legislative Ideas } \\ \text { Center } & \text { 100\% Life Kyiv region } \\ \text { CHESNO movement } & \text { CSO Mykolaiv Center for Journalist } \\ \text { CSO Kryvyi Rih Investigation Center } & \text { Investigations } \\ \text { CSO Center for Economic Strategy } & \text { CSO Ninth Pole } \\ \text { CSO Together Against Corruption } & \text { CSO Women's Anti-Corruption } \\ \text { CSO Agency for Urban Initiatives } & \text { Movement } \\ \text { CSO Unity and Development } & \text { CSO Center for Political Studies } \\ \text { Agency for Legislative Initiatives } & \text { CSO Electronic Democracy }\end{array}$

Ukrainian Arbitration Association

YouControl

CSO Zaporizhzhia Investigation Center

CSO Kharkiv Anti-Corruption Center Anti-Corruption Action Center 100\% Life Charity Fund CSO Centerfor Media Research Prozoro Public Control Platform August Virlich Foundation ZMINA Human Rights Center Center for Public Monitoring and Analysis

Public Oversight Council under the National Anti-Corruption Bureau Public Council at the National Agency on Corruption Prevention 


\title{
Instabiles Vertrauen: Warum Reformen die Glaubwürdigkeit des Geheimdiensts SBU bei der Öffentlichkeit kaum gestärkt haben
}

\author{
Von Eli C. Kaul (Kent State University)
}

DOI: $10.31205 /$ UA.243.02

\section{Zusammenfassung}

Dieser Artikel untersucht das Misstrauen in den Sicherheitsdienst der Ukraine (SBU) und beschreibt Gründe für das geringe öffentliche Vertrauen in ihn. Er macht deutlich, dass der Eindruck, der SBU sei korrupt und käme formal oder informell als Instrument zur Durchsetzung der Interessen der politischen und wirtschaftlichen Elite in der Ukraine zum Einsatz, auf das Erbe dieser Organisation zurückgeht, die zu Sowjetzeiten Teil des KGB war. Es wird gezeigt, wie sich das Ausmaß des Vertrauens in den SBU mit dessen Rolle im Rahmen wichtiger politischer Ereignisse und Skandale verändert und dass der Vertrauensmangel in höherem Maße systemischer Natur ist, als es auf den ersten Blick scheint.

\section{Einleitung}

Seit dem Euromaidan hat der Sicherheitsdienst der Ukraine sich um die Umsetzung zahlreicher Reformen bemüht. Diese Reformen waren sowohl auf westliche Ideale und Demokratisierungsbestrebungen der Ukraine als auch auf interne organisatorische Mängel bei der Bekämpfung der Separatisten im Donbas seit den Aufständen 2014 ausgerichtet. Außerdem ist der SBU selbst mit dem Erbe interner Kämpfe um systemische Korruption und Machtmissbrauch in Regierungssystem und Sicherheitsapparat (SA) der Ukraine beschäftigt. Zwischen diesen internen Auseinandersetzungen und den angestrebten Reformen besteht ein Konflikt, der verantwortlich ist für die fehlgeschlagenen Versuche, nach den Ereignissen des Maidans den Machtmissbrauch innerhalb des SBU zu beenden. Dabei gilt es allerdings festzuhalten, dass zahlreiche aktuelle und ehemalige SBU-Mitarbeiter sich mit diesem Zustand nicht zufriedengeben und die Benennung dieser organisatorischer Mängel befürworten. Allein das ist ein hoffnungsvolles Zeichen für eine Lösung des Problems von Korruption und Machtmissbrauch innerhalb des SBU.

Dieser Aufsatz analysiert mehrere Umfragen zu Vertrauen in Regierungs- und Nichtregierungsorganisationen in der Ukraine während der Zeit um die Orange Revolution (2004) und um den Euromaidan (2014). Zur Untersuchung des SBU interviewte der Autor 25 Experten, darunter aktuelle und ehemalige Mitarbeiter des SBU, Wissenschaftler, die sich mit dem SBU beschäftigen, ukrainische Journalisten und Politiker. Durch ergänzende Daten, die für ein anderes Projekt zur Erforschung der institutionellen Entwicklung des SBU erhoben wurden, lässt sich erklären, warum der SBU sein Ansehen in der ukrainischen Bevölkerung trotz zahlreicher Reformen seit den 2000er Jahren nicht verbessern konnte. Dazu zählen qualitative und quantitative Analysen aus Archivrecherchen sowie eine Inhaltsana- lyse von über 4.000 Medienberichten aus den Jahren 1991 bis 2018. Abschließend werden einige Vorschläge für systemische Reformen diskutiert, die den SBU befähigen könnten, seine zentralen Aufgaben besser auszuführen - und Vertrauen zu gewinnen.

\section{Ursprünge des Misstrauens gegenüber dem SBU}

In den turbulenten 1990er Jahren etablierten sich informelle Strukturen in der Organisationskultur des SBU (wie in den meisten politischen Organisationen der Ukraine). Diese Praktiken erwiesen sich als widerständig gegenüber formalen Reformen - rechtliche Schlupflöcher wurden ausgenutzt und Wege gefunden, um politischen Veränderungen ausweichen und Praktiken zur Bekämpfung der Korruption in der Ukraine umgehen zu können. Dazu zählte die Praxis des reiderstwo (Überfälle, bei denen Unternehmer mithilfe schwerer Waffen ihrer Eigentümer beraubt werden) sowie informelle Praktiken von einfacher Bestechung bis hin zu aufwendigen Verschleierungen illegaler Aktivitäten und Mord. Diese Praxis fiel zusammen mit der Entstehung der kryscha: "Dächer" informeller Organisationen von Kriminellen und Oligarchen, die Schutzgeld erpressten und illegale Dienste anboten, wie die Entsendung von Schlägertrupps, die mit Gewalt Lösungen erzwangen. Trotz der Entstehung dieser lukrativen informellen Praktiken schwankte die Zahl der SBU-Mitarbeiter in der Mitte der 1990er Jahre stark (siehe Grafik 1 auf S. 18) und konnte sich erst nach einer Reihe von Skandalen in den frühen 2000er Jahren erholen und stabilisieren.

Die Erneuerung der Personalsituation beim SBU in der zweiten Amtszeit von Präsident Kutschma lag begründet in der Sorge der Präsidialadministration um die Loyalität des SBU-Personals nach dem Gongadze-Mord und der Veröffentlichung der Melnytschenko-Tonbandaufnahmen (http://documents. 
worldbank.org/curated/en/982501468760201780/ Investigating-corruption-in-Ukraine-a-case-study-ofInternetjournalist-Georgy-Gongadze). Die Einstellung neuer Mitarbeiter verringerte den Einfluss jener SBUOffiziere, die als illoyal gegenüber Kutschma und seinen politischen Verbündeten galten - Andersdenkende in der Organisation wurden übertönt und neue konforme Mitarbeiter eingestellt. Die Administration reagierte auf solche Skandale also, indem sie hochrangige Offiziere austauschte, anstatt sich mit relevanten systemischen Faktoren zu befassen, etwa dem Fehlen einer internen Aufsichtsstelle zur Selbstkontrolle von SBU und Innenministerium (MWS).

Dass der Gongadze-Skandal nie komplett aufgearbeitet und abgeschlossen wurde, schadete dem Ansehen des SBU in der Kutschma-Ära, obwohl SBU-Chef Derkatsch und Innenminister Krawtschenko entlassen wurden. Die Skandale selbst stärkten die Opposition gegen Kutschma in der Rada, an anderen Stellen im Land kam es erneut zu Protesten und Kampfansagen gegen die Administration. Eine Reihe von Protesten von Oppositionsparteien - insbesondere der Ukrainischen Nationalversammlung - Ukrainische Nationale Selbstverteidigung (UNA-UNSO) und der Sozialistischen Partei der Ukraine - führte zur Entstehung der politischen Bewegung Ukraine ohne Kutschma. Es kam zu Zusammenstößen der Bewegung mit dem Innenministerium, bei denen der SBU Demonstranten festnahm. Anschließend zögerten die (von der Generalstaatsanwaltschaft beauftragten) Staatsanwälte die für die Klageerhebung nötigen Beweisaufnahmen hinaus, während die SBU-Abteilung zum Schutz von Beteiligten an Strafverfahren von ihrem Recht Gebrauch machte, Beschuldigte oder Zeugen »zu deren eigenem Schutz« zu verhaften und in Gewahrsam zu halten. Der über Formalitäten begründete Verzicht auf die Durchführung eines ordentlichen Prozesses wirkt bis heute nach und haftet etwa Versuchen zur Konfliktlösung mit den Separatisten im Donbas an, indem er zeigt, dass die ukrainischen Behörden durchaus geneigt sind, ihre Macht zu missbrauchen, um falsche Geständnisse zu erpressen oder Personen zu bestrafen, die nicht bereit sind, mit ihnen zu kooperieren.

\section{Belege für das Misstrauen gegenüber dem SBU}

2002 begannen die Stiftung Demokratische Initiativen und das Zentrum für Soziale und Politische Studien, Umfragen zu veröffentlichen, bei denen mehr als 1.200 repräsentativ ausgewählte Personen in sämtlichen Regionen der Ukraine befragt worden waren. Der Vergleich dieser Daten zeigt das veränderte Maß des Vertrauens der Öffentlichkeit zwischen 2002 und 2004 und ab 2005. Grafik 1 und 2 auf S. 16 zeigen das Vertrauen in jede einzelne Institution. In den Grafiken 3 und 4 auf S. 17 wurden Gesamtwerte für verschiedene Arten von Institutionen generiert, unter denen die einzelnen Organisationen zusammengefasst sind. Diese Werte weisen das allgemeine Vertrauen in Nichtregierungsorganisationen, in Regierungsorganisationen (ohne die Organisationen des Sicherheitsapparats), in die für die Sicherheit der Ukraine zuständigen Institutionen und in die Medien aus. Die Kirche und die Gewerkschaften zählen als Nichtregierungsorganisationen. Zu den Regierungsorganisationen gehören Gerichte, lokale Verwaltungen, die Werchowna Rada, der Präsident und das Ministerkabinett. Die Sicherheitsbehörden sind Generalstaatsanwaltschaft, Armee, SBU, Polizei und in den späteren Jahren auch das Nationale Antikorruptionsbüro der Ukraine (NABU).

Grafik 1 enthält Daten über das Vertrauen der Öffentlichkeit im Jahr 2002. Diese zeigen, dass 44 Prozent der Bevölkerung Kutschma im Großen und Ganzen vertrauten. Polizei und Ministerkabinett sprachen jeweils etwa 38 Prozent der Befragten ihr Vertrauen aus. Dem SBU wurde deutlich weniger vertraut - nur etwas unter 20 Prozent gaben an, dieser Organisation zumindest in gewissem Maße zu vertrauen. Grafik 2 zeigt anhand von Daten aus dem Jahr 2004, dass durch die Ereignisse nach den Skandalen um Gongadze und Krawtschenko sehr viel Vertrauen verloren gegangen ist. Der SBU gewann bei der Öffentlichkeit an Vertrauen und erreichte einen Wert von 23 Prozent, während Kutschma viel öffentliches Vertrauen verlor - seine Werte sanken von 44 auf nur noch etwas über zehn Prozent im Juli 2004. Das Vertrauen in Ministerkabinett und Polizei sank ebenfalls stark, von je 38 auf 9,5 bzw. 13,7 Prozent. Der SBU handhabte die genannten Skandale gut - sein Vorgehen und seine Äußerungen in der Kontroverse bewirkten eine leichte Steigerung des Vertrauens in die gesamte Behörde. Den Sicherheitsapparat als Ganzes beschädigten die Skandale dagegen, wie der Vergleich ihrer in Grafik 3 ausgewiesenen Vertrauenswerte zeigt. Diese Vertrauenswerte bilden den Beginn der Veränderung des Verhältnisses des SBU zur Gesellschaft im Allgemeinen ab. Er agiert zwar nach wie vor als politisierter Sicherheitsdienst, seine politische Ausrichtung scheint jedoch stärker auf konkurrierende Elitenverbände, Oligarchen und die Organisierte Kriminalität ausgerichtet als auf den Erhalt der Kontrolle über die Öffentlichkeit. Diese Aufgabe übernehmen zunehmend das Innenministerium und lokale Polizei.

Die Sonderstellung des SBU, der als einzige Organisation des ukrainischen Sicherheitsapparates direkt dem Büro des Präsidenten unterstellt ist und vom Parlament nur in sehr geringem Maße kontrolliert werden kann, macht ihn zu einem unverzichtbaren Instrument der politischen Kontrolle. Direkt nach seinem Sieg bei 
der Präsidentschaftswahl 2004 kritisierte Juschtschenko die SBU-Praxis der verdeckten Beobachtung des politischen und wirtschaftlichen Umfelds noch scharf. Erst als er Schwierigkeiten hatte, mit den von ihm erwünschten Methoden zum Erfolg zu kommen, erachtete Juschtschenko diese Methode als sinnvoll für die Überwachung der politischen Landschaft der Ukraine. Bis heute ist es deshalb unter politischen Persönlichkeiten, Aktivisten und Geschäftsleuten nicht unüblich, sich zur Besprechung potenziell sensibler Informationen nicht in den eigenen Büros zu treffen, sondern stattdessen alternative Orte aufzusuchen.

Petro Poroschenko griff bei seinen Bemühungen, eine Antikorruptionsreform innerhalb des Sicherheitsapparats durchzuführen, zunächst auf Methoden aus der Juschtschenko-Ära zurück: Der Apparat wurde durch eine Aussortierung des mit der vorangegangenen Administration loyalen Personals gesäubert. Das 2015 gegründete unabhängige Nationale Antikorruptionsbüro der Ukraine (NABU) sollte die Korruption in den hohen Rängen ins Visier nehmen und schien dabei anfänglich einige Erfolge zu erzielen. Die Eingliederung des verhältnismäßig kleinen NABU in die Struktur des ukrainischen Sicherheitsapparats war jedoch nicht ideal - das NABU verfügt über insgesamt knapp 700 Mitarbeiter, im SBU arbeiten dagegen 31.000 und im Innenministerium 151.000 Mitarbeiter.

In der frühen Reformperiode nach dem Maidan leitete Poroschenko Maßnahmen ein, um SBU und Sicherheitsapparat von korrupten Beamten der vorherigen Administration zu säubern. Anders als unter den vorangegangenen Administrationen gab es zu dieser Zeit allerdings einen eskalierenden Konflikt im Donbas und die Autonome Republik Krim stand nun unter russischer Kontrolle. Das beschränkte die »Optionen zur personellen Veränderung des SBU, denn wir mussten die Separatisten bekämpfen ... Er [Poroschenko] arbeitete mit dem Westen zusammen und gründete das NABU, aber er konnte nicht alle korrupten SBUler entlassen" (Interview mit einem Beamten). $\mathrm{Zu}$ dieser Zeit stagnierte die Entwicklung des SBU. Die Eingliederung des NABU hinderte das SBU-Direktorium an der Ausführung seiner Hauptaufgabe - der Bekämpfung von Organisierter Kriminalität und Korruption -, indem etliche Direktoriumsmitglieder 2014/2015 ins NABU versetzt wurden (Interview mit einem politischen Amtsträger). Die fehlende Glaubwürdigkeit von Poroschenkos Reformen bei der Öffentlichkeit wurde in dieser Zeit immer wieder deutlich und der SBU »konzentrierte sich in erster Linie auf Terrorismus und terroristische Bedrohungen durch kriminelle Gruppen und oppositionelle Oligarchen« (Interview mit einer Person aus der Politik). So wird die andauernde Politisierung des SBU durch die Administration möglich, die die- sen funktionalisiert, um ihre Ziele durch die selektive Anwendung ihrer Befugnisse zu erreichen.

Grafik 4 (S. 17) zeigt das öffentliche Vertrauen in verschiedene Arten von Regierungsorganisationen im Jahr 2014, bevor Poroschenko ins Präsidentenamt gewählt wurde. Das Vertrauen in Regierungs- und Sicherheitsapparat liegt zu diesem Zeitpunkt bei jeweils etwa 50 Prozent. Im Dezember 2018, nach vier Jahren Amtszeit Poroschenkos, belegt eine andere Umfrage einen drastischen Verlust an öffentlichem Vertrauen: Das Vertrauen in Regierungsinstitutionen in Poroschenkos Amtszeit ist auf neun Prozent gefallen; das Vertrauen in die Sicherheitsinstitutionen auf 37 Prozent. Dies lag vor allem an den informellen Praktiken der Korruption, denn, so erklärte ein Beamter in einem Interview:

»das Volk weiß, dass viele Amtsträger in SBU und beim Innenministerium den Interessen von Oligarchen zuarbeiten, und der Präsident ist selbst ein Oligarch".

Der Widerstand gegen Reformen ist nicht der einzige Grund für die fest verankerten korrupten Strukturen im SBU, denn »solche Reformen brauchen Zeit, um umgesetzt zu werden ... und bis zu dem Moment, an dem diese Reformen offiziell in Kraft treten, haben die korrupten Beamten schon Wege gefunden, um sie zu umgehen und ihre Geschäfte weiterzuführen« (Interview mit einem Politikexperten). Im Zuge der Gründung des NABU hat das SBU nicht nur viele seiner Aufgaben im Bereich Korruptionsbekämpfung verloren, sondern auch viel öffentliches Vertrauen. Grafik 5 (S. 18) zeigt das Maß an Vertrauen, das 2018 in die jeweiligen Sicherheitsorgane gesetzt wurde: Dem NABU wird mit 72 Prozent am meisten Vertrauen entgegengebracht, der Armee mit 53 Prozent zwar deutlich weniger, aber wesentlich mehr als dem SBU und der Polizei, denen rund 25 Prozent vertrauten. In der Gründung des NABU zeigt sich das Bedürfnis der ukrainischen Öffentlichkeit nach einem Ende der Korruption. SBU und Innenministerium wird dagegen nicht zugetraut, ihre Aufgaben ohne Skandale ausführen zu können, so dass das Vertrauen in diese Institutionen niedrig ist.

\section{Fazit}

Für die Entwicklung der Demokratie ist die Entstehung öffentlichen Vertrauens in die Sicherheitsdienste von zentraler Bedeutung. Im Fall der Ukraine verhinderten das Erbe des KGB sowie das Chaos und die Korruption, die in den 1990er Jahren um sich griffen, dass ein derartiges Vertrauen entstehen konnte. Das SBU und die anderen ukrainischen Sicherheitsorganisationen galten vielmehr als Instrumente zur Kontrolle der Macht von mächtigen Eliten wie Oligarchen und politischen Akteuren mit Verbindungen zur Organisierten Kriminalität. Ein großer Teil der SBU-Aktivitäten in den 1990er 
und 2000er Jahren rechtfertigte dieses Bild und führte zu einem andauernden Misstrauen gegenüber dem SBU. Gleichzeitig äußerten die meisten Interviewpartner die Beobachtung, dass es innerhalb des SBU viele Einzelpersonen gebe, die die Lebensqualität und die Sicherheit der ukrainischen Bürger verbessern wollen. Angesichts der hierarchischen Top-down-Struktur des SBU sei es für diese Personen jedoch schwer, darauf hinzuwirken. Hier wird ein systemisches Problem innerhalb der SBU-Struktur sichtbar, das verhindert, dass einzelne Personen aktiv werden, wenn sie Zeugen von Machtmissbrauch und Korruption werden. Außerdem gibt es Befürchtungen, dass Teile des SBU Verbindungen zu ausländischen - namentlich russischen - Geheimdiensten unterhalten, was mit Blick auf den Konflikt im Donbas und die Annexion der Krim das Misstrauen gegenüber dem SBU zusätzlich verstärkt.

Die Methode der verdeckten Überwachung, die beim SBU weiterhin Anwendung findet, ist in den systemischen Strukturen des ukrainischen Sicherheitsapparats, im politischen System und in den formalen und informellen Wirtschaftsstrukturen derzeit fest verankert. Dass verdeckte Überwachung stattfinden kann, schmälert das Vertrauen in der ukrainischen Gesellschaft. Viele Menschen begleitet, während sie ihrem Alltag nachgehen, bewusst oder unbewusst die Vorstellung, ihre Handlungen würden durch den SBU beobachtet. Außerdem besteht der Eindruck, der SBU diene den Interessen der Politik, und der SBU komme vor allem zum Einsatz, um die Sicherheit der Eliten zu schützen - und nicht die Bevölkerung. Deshalb liegen die Vertrauenswerte des SBU in der Bevölkerung lediglich bei 25 Prozent.

Der effizienteste Schritt, um das öffentliche Vertrauen in den SBU zu steigern, wäre die Errichtung von Abteilungen zur Selbstkontrolle innerhalb der eigenen SBU-Struktur. Eine solche "Abteilung für innere Angelegenheiten« sollte die Tätigkeiten des SBU-Personals sämtlicher Bereiche überwachen und damit - zumindest vordergründig - eine Atmosphäre schaffen, in der illegale Aktivitäten ans Licht kommen und geahndet werden. Diese Lösung stößt an ihre Grenzen, da sie das Potenzial für interne Korruption in sich trägt. Nötig sind also auch Alternativen, um die Entstehung von öffentlichem Vertrauen zu ermöglichen. Die einzige wirkliche Lösung für das gesunkene Vertrauen in den SBU wäre es, Oligarchen und Politiker, die in Korruption verwickelt sind, effektiv zu verfolgen - und nicht nur diejenigen, die gegen die derzeitige politische Führung opponieren. Ob der SBU jemals versuchen wird, mehr Vertrauen bei der ukrainischen Gesellschaft zu gewinnen, bleibt abzuwarten. Beginnt er mit der Untersuchung und Bestrafung illegaler Aktivitäten nicht bei seinem eigenen Personal, wird das in ihn gesetzte öffentliche Vertrauen wohl gering bleiben.

Übersetzung aus dem Englischen von Sophie Hellgardt

\section{Über den Autor}

Eli C. Kaul ist Doktorand der Politikwissenschaften an der Kent State University. Derzeit bereitet er die Verteidigung seiner Dissertation "The Evolution of the Security Services of Ukraine: Institutional Change in the Post-Soviet Security Apparatus« vor. Seine Forschungsschwerpunkte sind Korruption und Sicherheitspolitik mit Schwerpunkt auf die Länder der früheren Sowjetunion.

\section{Hinweis}

Für diese Untersuchung wurden sechs aktuelle Angehörige des ukrainischen Sicherheitsdiensts interviewt (drei arbeiteten für den SBU, zwei für das Innenministerium, einer bei den Ukrainischen Streitkräften), drei ehemalige Angehörige des SBU, sieben politische Amtsträger (drei von ihnen gewählt, vier ernannt), sieben auf den ukrainischen Sicherheitsapparat spezialisierte Wissenschaftler und drei Journalisten, die sich regelmäßig mit dem SBU befassen. Die Interviews dauerten zwischen 45 Minuten und zwei Stunden und fanden als persönliche Treffen statt. Zu ihrer eigenen Sicherheit bleiben alle Interviewpartner anonym. Thematisiert wurden die SBU-Reformen, die Politisierung des SBU unter verschiedenen Präsidenten und Verbindungen des SBU zu informellen Praxen. Das Thema Korruption kam in allen Interviews zur Sprache, bis auf einen Interviewten betrachteten sämtliche Gesprächspartner Korruption als große Schwachstelle des SBU wie auch des gesamten politischen Systems der Ukraine.

\section{Lesetipps}

- Bennett, Gordon. 2004. The Sbu: The Security Service of Ukraine. Camberley, Surrey: Conflict Studies Research Center.

- Fisun, Oleksandr. 2015. “The Future of Ukraine's Neopatrimonial Democracy.” PonarsEuarasia - Policy Memos. http://www.ponarseurasia.org/memo/future-ukraine-neopatrimonial-democracy (June 3, 2017).

- Galeotti, M. 2005. 'The Russian 'Mafiya': Consolidation and Globalisation', in: M. Galeotti (ed.), Global Crime Today: The Changing Face of Organised Crime New York and London: Routledge : 54-70. 
- Koshiw, J. V. 2013. Abuse of Power - Corruption in the Office of the President. Artemia Press Limited.

- Kuzio, Taras. 2015. Ukraine: Democratization, Corruption, and the New Russian Imperialism. Santa Barbara, California: Praeger.

- Marten, Kimberly. 2015. “The Security Costs and Benefits of Non-State Militias: The Example of Eastern Ukraine.” PonarsEuarasia - Policy Memos. http://www.ponarseurasia.org/memo/security-costs-andbenefits-non-state-militiasexample-eastern-ukraine (June 3, 2017).

- Rojansky, M. 2014. 'Corporate Raiding in Ukraine: Causes, Methods and Consequences', Demokratizatsiya: The Journal of Post-Soviet Democratization, 22, 3.

- Shabunin, V., Shcherban, O. 2020. "The reform of the SBU: neither here nor there”. https://antac.org.ua/en/news/ the-reform-of-the-sbu-neither-here-nor-there/.

\section{Vertrauen in den Sicherheitsdienst}

Tabelle 1: Vertrauen in Institutionen*

\begin{tabular}{|c|c|c|c|c|c|c|}
\hline Institution & 2002 & 2004 & 2005 & 2014 & 2015 & 2018 \\
\hline Armee & 28,8 & 30,6 & 59 & 58,9 & & 53,1 \\
\hline $\begin{array}{l}\text { Sicherheitsdienst der Ukraine } \\
\text { (SBU) }\end{array}$ & 19,9 & 23,4 & 44 & 43,7 & 34 & 24,2 \\
\hline $\begin{array}{l}\text { Nationales Antikorruptions- } \\
\text { büro (NABU) }\end{array}$ & & & & & & 72,1 \\
\hline Generalstaatsanwaltschaft & & 14,3 & 35 & & 28,2 & 11,2 \\
\hline Polizei & 38 & 13,7 & 37 & & & 25,3 \\
\hline Justiz & 27,6 & 13,8 & 35 & & 28,2 & 6 \\
\hline Lokale Verwaltung & 24 & 14,4 & 37 & & & \\
\hline Lokale Räte & 23 & 15,1 & 41 & & & \\
\hline Präsident & 44 & 10,7 & 60 & 52 & & 12,3 \\
\hline Parlament & & 5,7 & 39 & 48 & & 5,9 \\
\hline Ministerkabinett & 38 & 9,5 & 46 & 52 & & \\
\hline Kirche & 47,1 & 50,8 & 47 & 65,6 & & 56,9 \\
\hline Gewerkschaften & 26,4 & 13,1 & 29 & & & \\
\hline Medien & 18,7 & 17,4 & 64,6 & 39,6 & & 29,2 \\
\hline \multicolumn{7}{|l|}{ Gruppiert: } \\
\hline Sicherheitsdienst & 28,9 & 20,5 & 43,8 & 51,3 & 31,1 & 37,18 \\
\hline Staatliche Institutionen & 31,3 & 12,7 & 43,8 & 52 & 28,2 & 9,15 \\
\hline Nichtstaatliche Institutionen & 36,7 & 31,9 & 38 & 65,6 & & 56,9 \\
\hline
\end{tabular}

*Anmerkung: Die Zahlen setzen sich zusammen aus verschiedenen Umfragen mehrerer Umfrageinstitute und stellen den jährlichen Mittelwert dar. Quelle: Eigene Zusammenstellung des Autors. 
Grafik 1: Institutionenvertrauen 2002

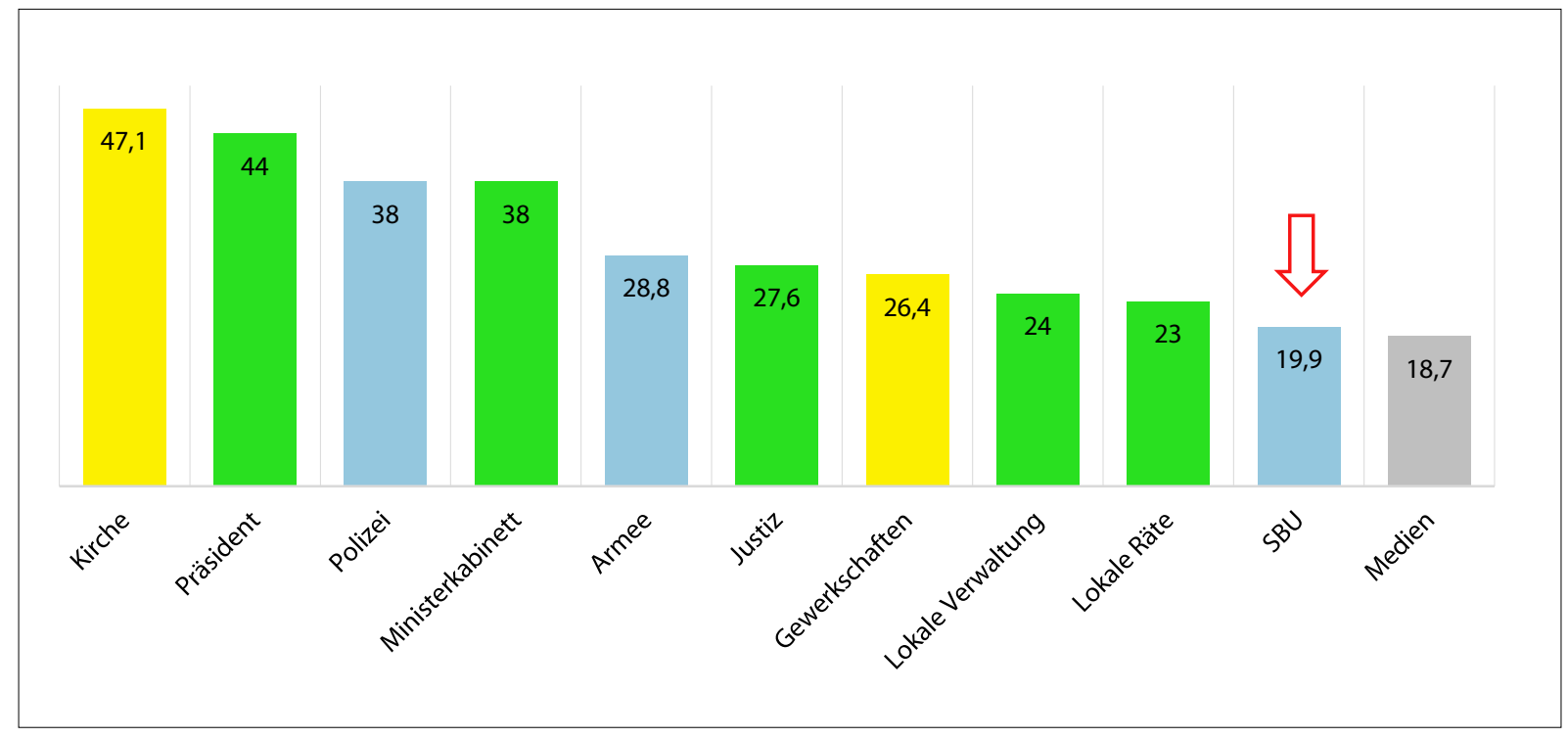

*Anmerkung: Die Zahlen setzen sich zusammen aus verschiedenen Umfragen mehrerer Umfrageinstitute und stellen den jährlichen Mittelwert dar. Quelle: Eigene Zusammenstellung des Autors.

\section{Grafik 2: Institutionenvertrauen 2004}

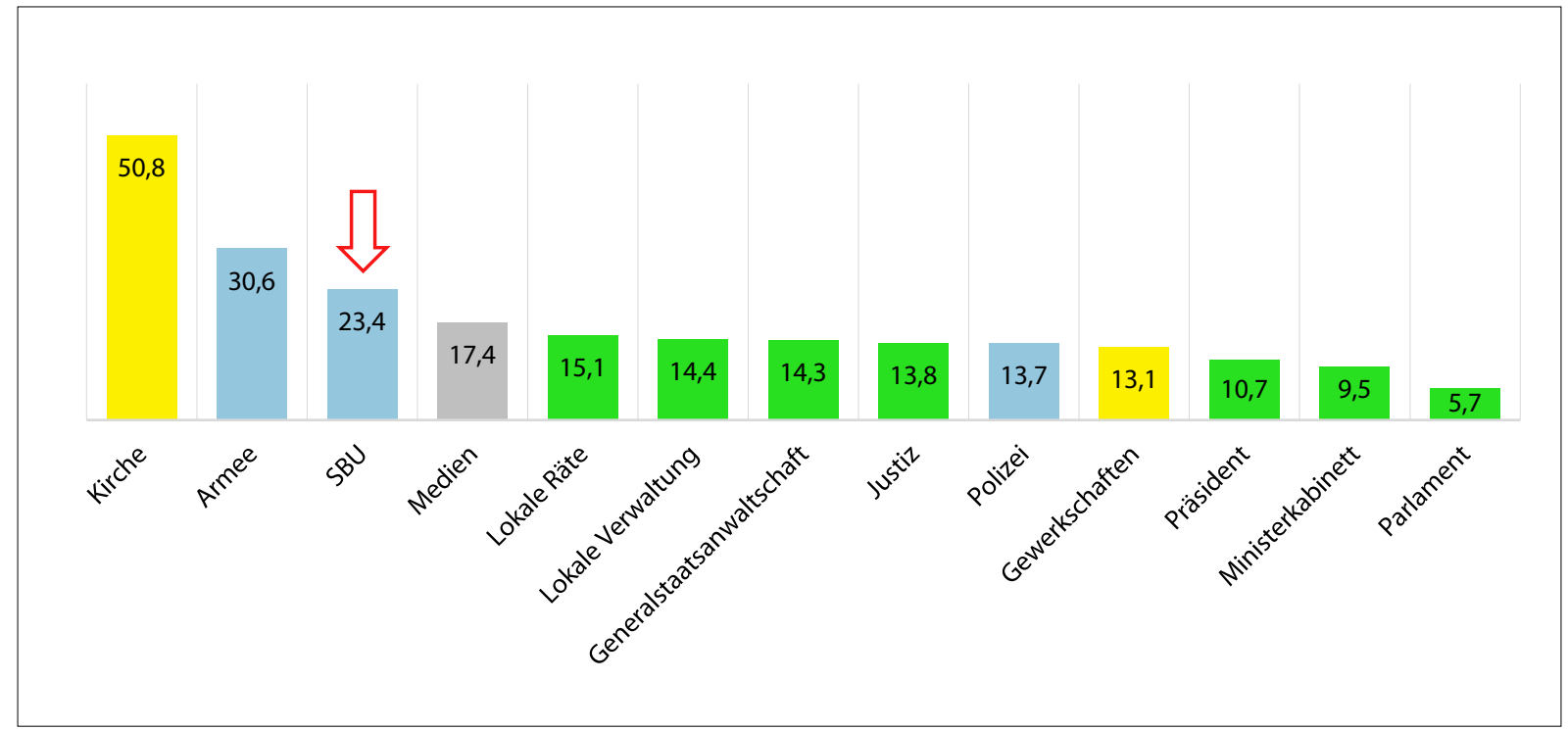

*Anmerkung: Die Zahlen setzen sich zusammen aus verschiedenen Umfragen mehrerer Umfrageinstitute und stellen den jährlichen Mittelwert dar. Quelle: Eigene Zusammenstellung des Autors. 


\section{Grafik 3: Vertrauen 2002 und 2004 (gruppiert)}

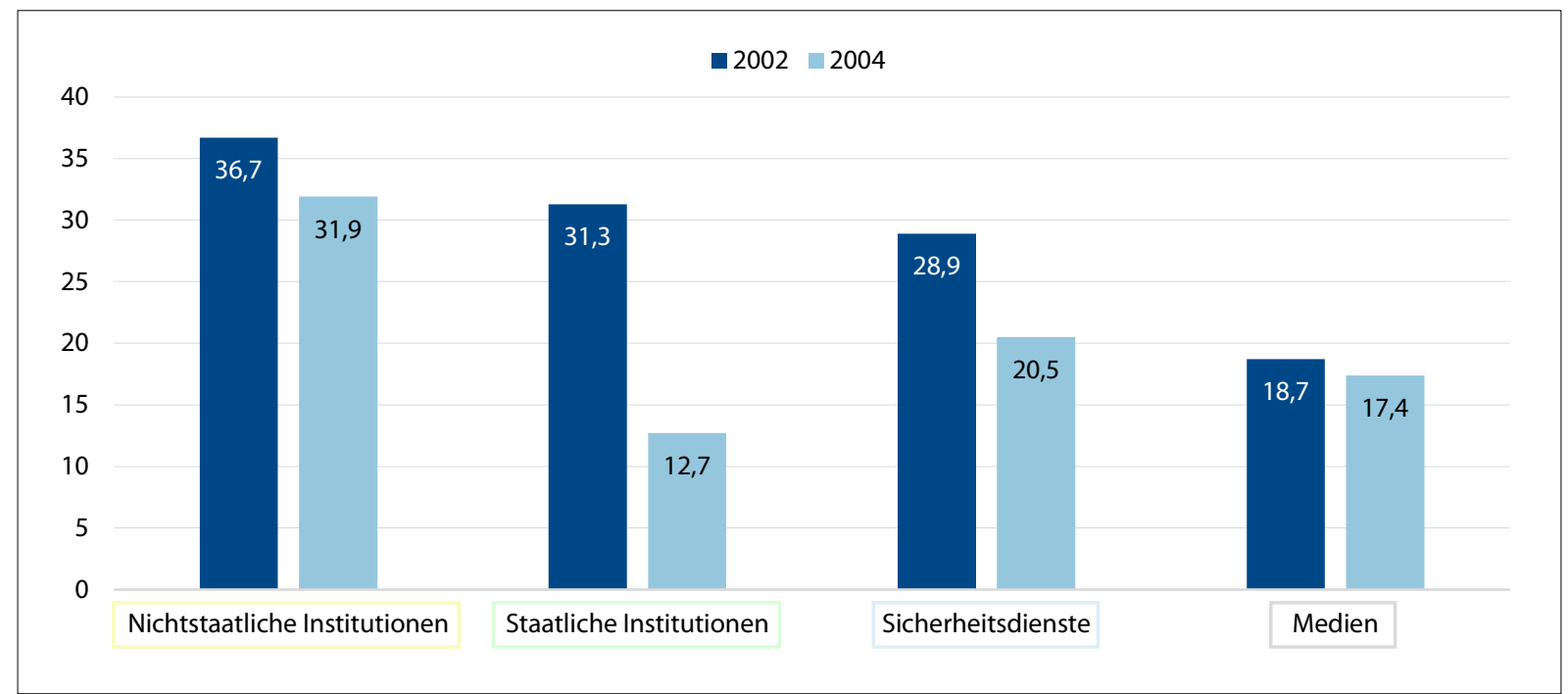

*Anmerkung: Die Zahlen setzen sich zusammen aus verschiedenen Umfragen mehrerer Umfrageinstitute und stellen den jährlichen Mittelwert dar. Quelle: Eigene Zusammenstellung des Autors.

\section{Grafik 4: Vertrauen 2014 und 2018 (gruppiert)}

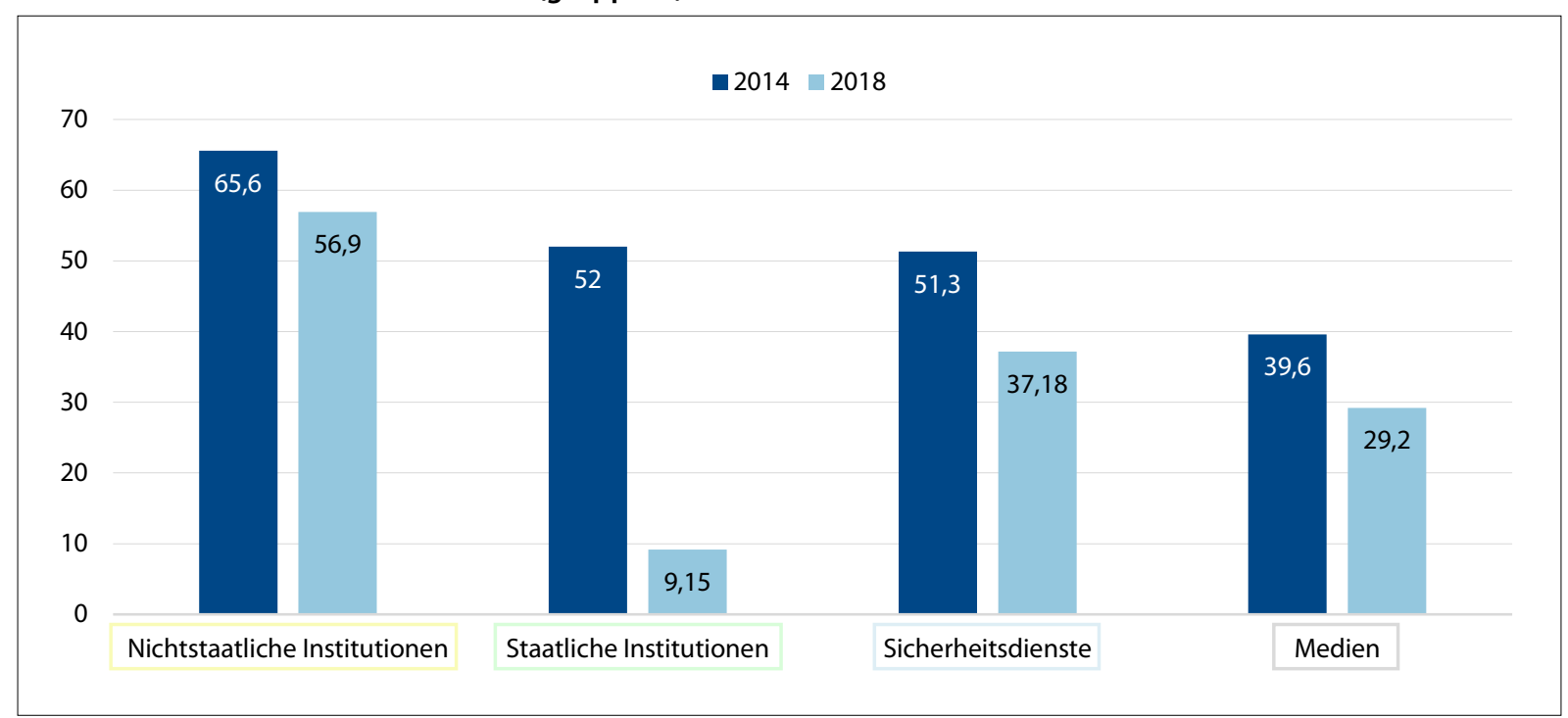

*Anmerkung:Die Zahlen setzen sich zusammen aus verschiedenen Umfragen mehrerer Umfrageinstitute und stellen den jährlichen Mittelwert dar. Quelle: Eigene Zusammenstellung des Autors. 
Grafik 5: Institutionenvertrauen 2018

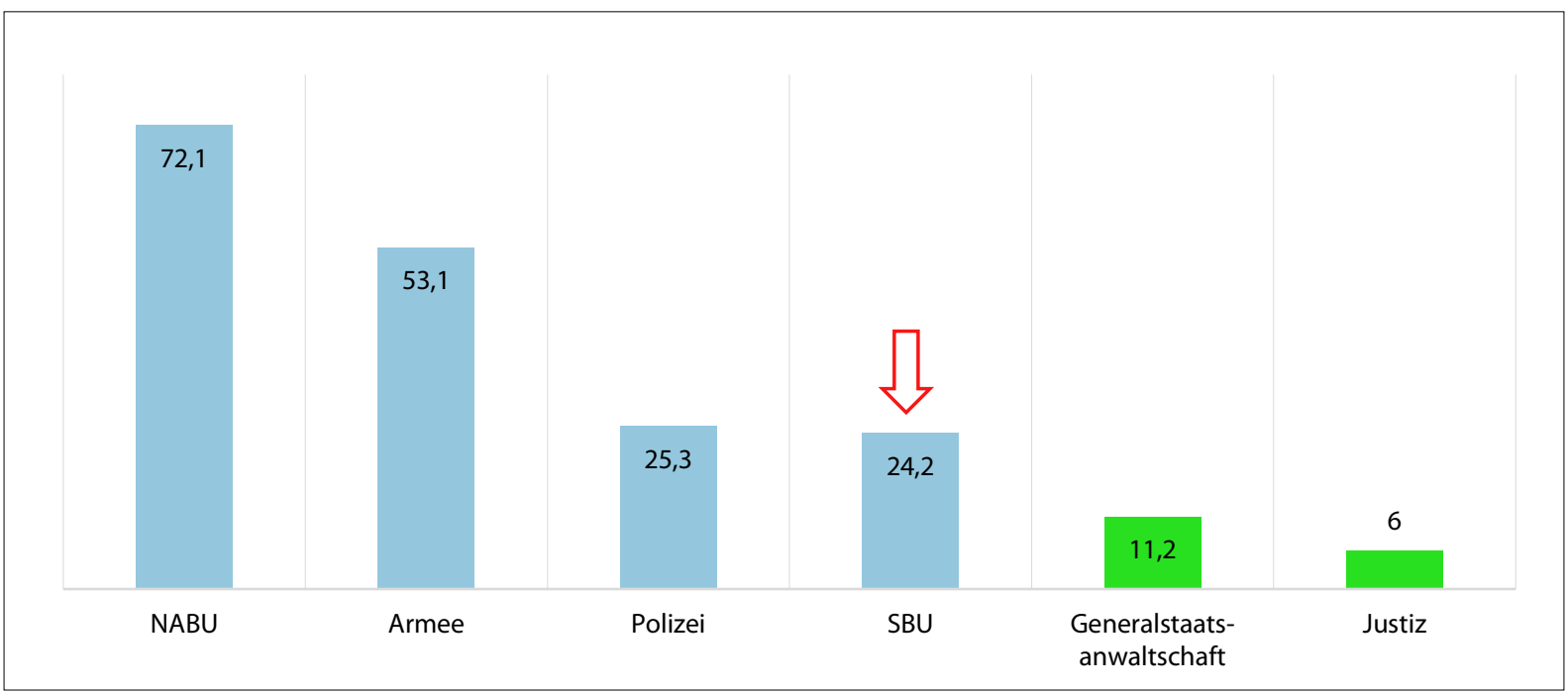

*Anmerkung: Die Zahlen setzen sich zusammen aus verschiedenen Umfragen mehrerer Umfrageinstitute und stellen den jährlichen Mittelwert dar. Quelle: Eigene Zusammenstellung des Autors.

STATISTIK

\section{Personalstärke des SBU}

Grafik 1: Personalstärke des SBU 1991 - 2004

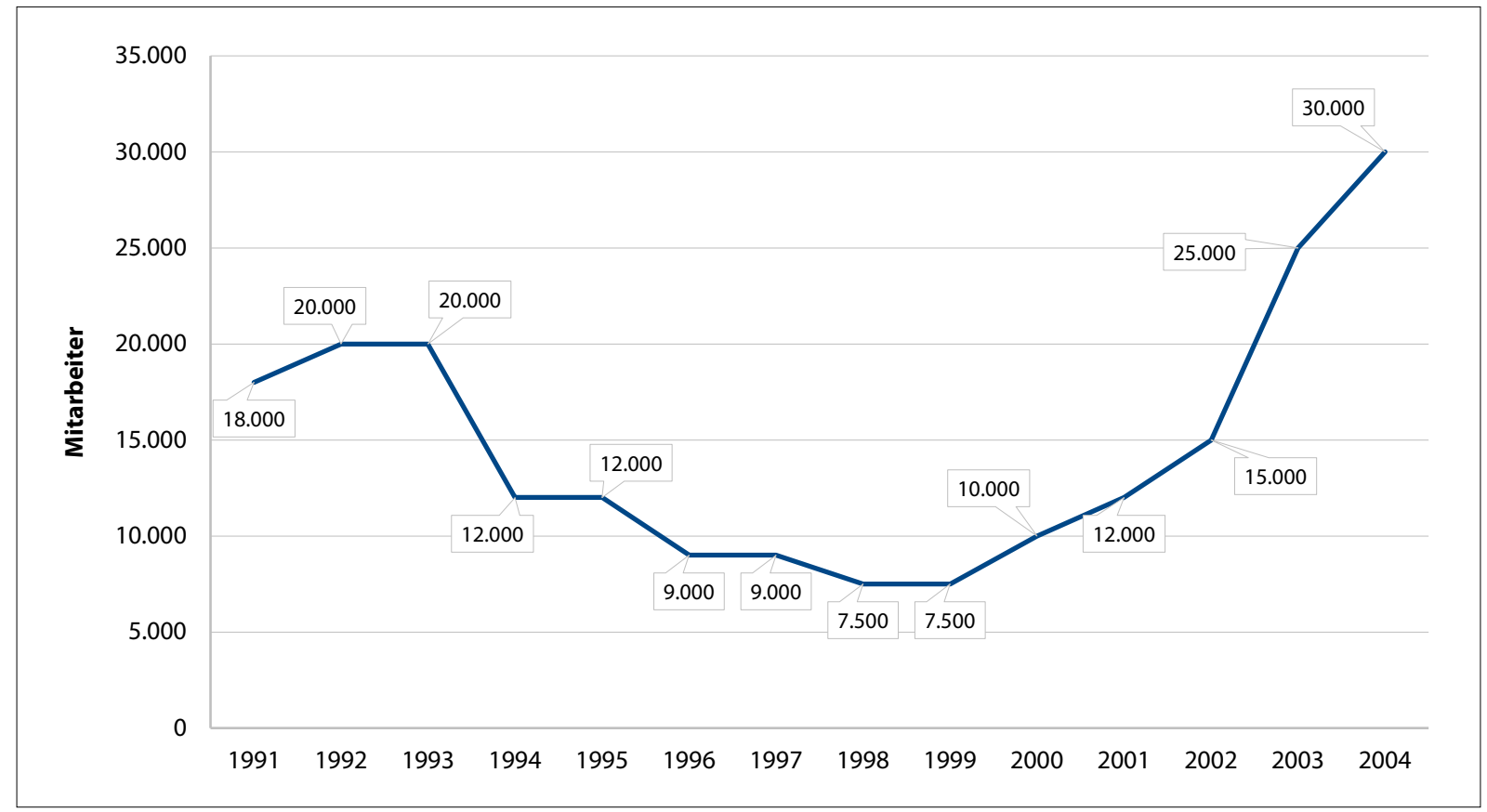

Quelle: Eigene Berechnungen des Autors basierend auf Interviewaussagen. 


\section{Die Verbreitung von Covid-19 in der Ukraine}

Grafik 1: Ausbreitung von Covid-19 im Vergleich (Fälle pro 100.000 Einwohner, 1. März 27. November 2020)

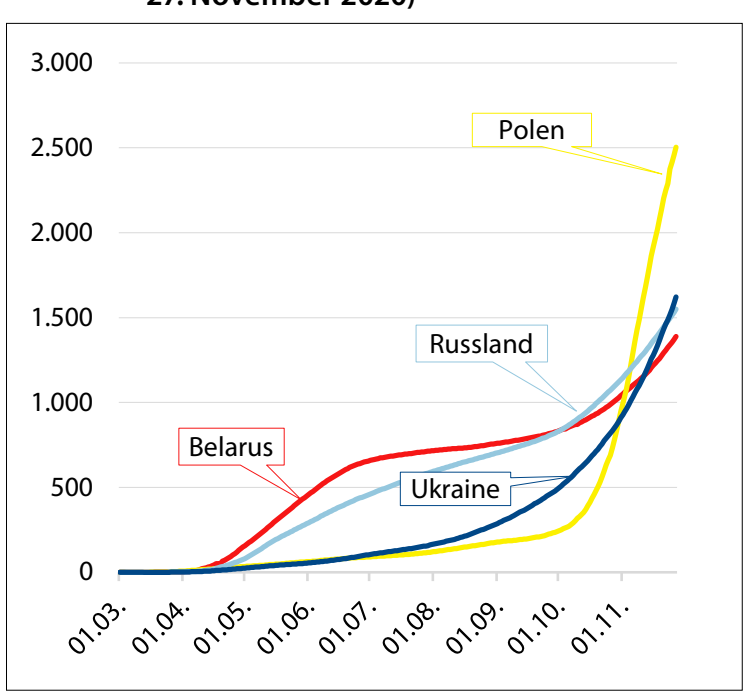

Tabelle 1: Ausbreitung von Covid-19 im Vergleich (Fälle, 16. - 27. November 2020)

\begin{tabular}{|c|c|c|c|c|}
\hline Datum & Belarus & Polen & Russland & Ukraine \\
\hline 16.11. & 115.448 & 733.788 & 1.932 .711 & 561.581 \\
17.11. & 116.699 & 752.940 & 1.954 .912 & 573.758 \\
18.11. & 118.008 & 772.823 & 1.975 .629 & 586.522 \\
19.11. & 119.390 & 796.798 & 1.998 .966 & 600.152 \\
\hline 20.11. & 120.847 & 819.262 & 2.023 .025 & 614.986 \\
21.11. & 122.435 & 843.475 & 2.047 .563 & 629.850 \\
22.11. & 123.999 & 861.331 & 2.071 .858 & 642.215 \\
\hline 23.11. & 125.482 & 876.333 & 2.096 .749 & 653.442 \\
\hline 24.11. & 126.953 & 909.066 & 2.120 .836 & 665.968 \\
\hline 25.11. & 128.449 & 924.422 & 2.144 .229 & 680.132 \\
\hline 26.11. & 130.012 & 941.112 & 2.169 .424 & 695.755 \\
\hline 27.11. & 131.633 & 958.416 & 2.196 .691 & 712.249 \\
\hline
\end{tabular}

Für die Zahlen vom 01.03.-15.11.2020 siehe die Statistik »Die Verbreitung von Covid-19 in der Ukraine« in den Ukraine-Analysen $233-242$.

Auch wenn die Zahlen alle aus einer Quelle stammen, sind sie nur begrenzt vergleichbar, da sich zwischen den Ländern der Umfang der Tests und die Erfassung von Covid-19 als Todesursache teilweise deutlich unterscheiden.

Quelle: Johns Hopkins Universität. Stand: 27.11.2020, 20:27 Uhr MESZ https://oronavirus.jhu.edu/map.html; https://github.com/CSSEGISandData/COVID-19/ blob/master/csse_covid_19_data/csse_covid_19_time_series/time_series_covid19_confirmed_global.csv; Einwohnerzahlen: CIA World Factbook, https:// www.cia.gov/library/publications/the-world-factbook/

Grafik 2: Todesfälle durch Covid-19 im Vergleich (Todesfälle pro 100.000 Einwohner, 1. März - 27. November 2020)

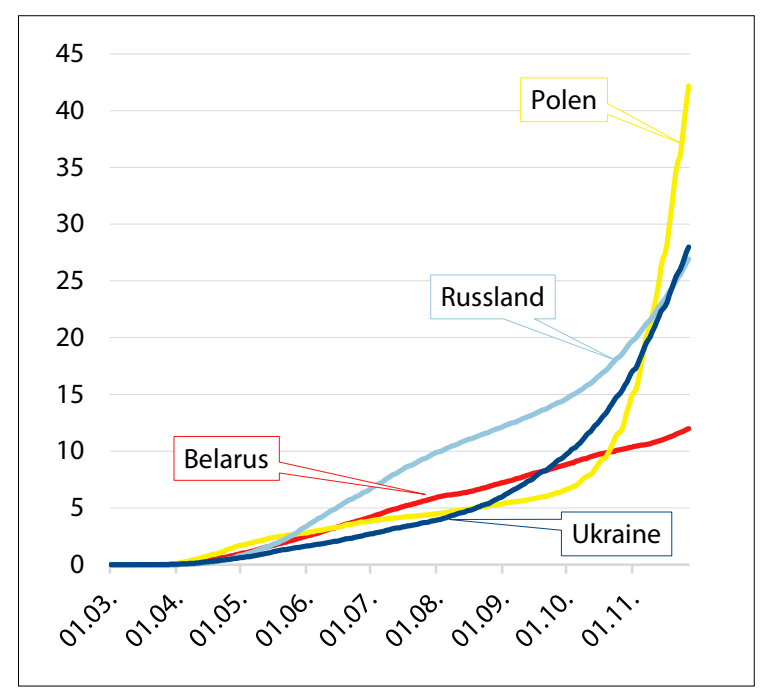

Tabelle 2: Todesfälle durch Covid-19 im Vergleich (16. - 27. November 2020)

\begin{tabular}{|c|c|c|c|c|}
\hline Datum & Belarus & Polen & Russland & Ukraine \\
\hline 16.11. & 1.053 & 10.491 & 33.184 & 10.002 \\
\hline 17.11. & 1.060 & 10.848 & 33.619 & 10.168 \\
18.11. & 1.067 & 11.451 & 34.068 & 10.431 \\
19.11. & 1.074 & 12.088 & 34.525 & 10.694 \\
\hline 20.11. & 1.081 & 12.714 & 34.980 & 10.929 \\
\hline 21.11. & 1.089 & 13.288 & 35.442 & 11.149 \\
\hline 22.11. & 1.096 & 13.618 & 35.838 & 11.292 \\
\hline 23.11. & 1.104 & 13.774 & 36.192 & 11.423 \\
\hline 24.11. & 1.112 & 14.314 & 36.675 & 11.619 \\
\hline 25.11. & 1.119 & 14.988 & 37.173 & 11.857 \\
\hline 26.11. & 1.128 & 15.568 & 37.688 & 12.091 \\
\hline 27.11. & 1.136 & 16.147 & 38.175 & 12.292 \\
\hline
\end{tabular}

Für die Zahlen vom 01.03.-27.11.2020 siehe die Statistik »Die Verbreitung von Covid-19 in der Ukraine« in den Ukraine-Analysen $233-242$.

Auch wenn die Zahlen alle aus einer Quelle stammen, sind sie nur begrenzt vergleichbar, da sich zwischen den Ländern der Umfang der Tests und die Erfassung von Covid-19 als Todesursache teilweise deutlich unterscheiden.

Quelle: Johns Hopkins Universität. Stand: 27.11.2020 Uhr 20:27 MESZ https://coronavirus.jhu.edu/map.html; https://github.com/CSSEGISandData/COVID-19/ blob/master/csse_covid_19_data/csse_covid_19_time_series/time_series_covid19_deaths_global.csv; Einwohnerzahlen:CIAWorld Factbook, https://www. cia.gov/library/publications/the-world-factbook/ 
Grafik 3: Regionale Verteilung der Covid-19-Infektionen

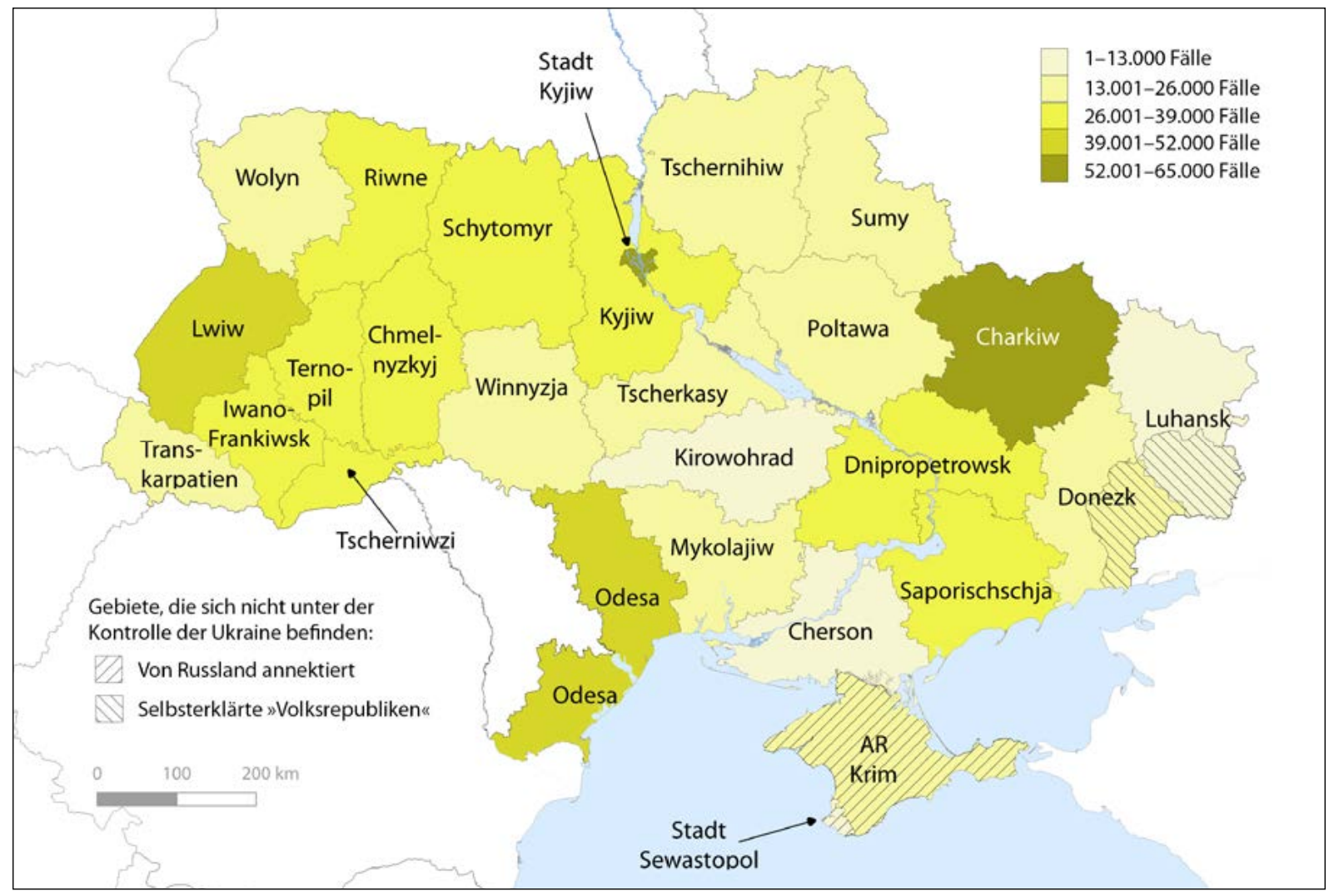

\begin{tabular}{|l|r|r|}
\hline \multicolumn{1}{|c|}{ Region } & Fälle & Todesfälle \\
\hline Charkiw & 53.433 & 730 \\
\hline Cherson & 9.345 & 187 \\
\hline Chmelnyzkyj & 27.703 & 421 \\
\hline Dnipropetrowsk & 33.115 & 823 \\
\hline Donezk & 21.344 & 381 \\
\hline Iwano-Frankiwsk & 34.045 & 597 \\
\hline Kiew & 34.241 & 652 \\
\hline Stadt Kiew & 64.019 & 1.227 \\
\hline Kirowohrad & 4.706 & 146 \\
\hline Luhansk & 5.925 & 154 \\
\hline Lwiw & 43.611 & 1.195 \\
\hline Mykolajiw & 16.731 & 289 \\
Odesa & 40.535 & 536 \\
Poltawa & 20.204 & 425 \\
Riwne & 29.320 & 369 \\
Saporischschja & 27.226 & 282 \\
\hline Schytomyr & 27.926 & 476 \\
Sumy & 25.662 & 348 \\
\hline Ternopil & 26.752 & 368 \\
Tscherkassy & 20.668 & 180 \\
\hline Tschernihiw & 16.485 & 91 \\
Tscherniwzi & 32.492 & 623 \\
\hline Transkarpatien & 22.425 & 518 \\
Winnyzja & 16.370 & 281 \\
\hline Wolyn & 22.906 & 418 \\
\hline AR Krim* & 14.468 & 264 \\
Stadt Sewastopol* & 4.098 & 112 \\
\hline
\end{tabular}

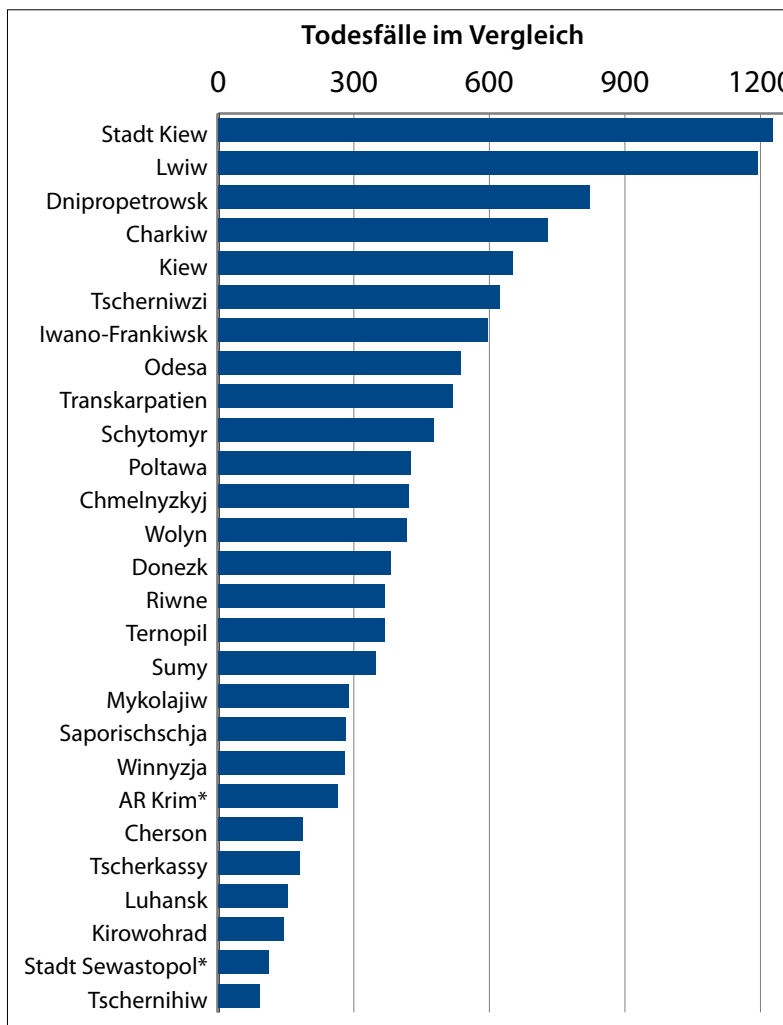

* inoffizielle Daten; Datenquelle: https://www.pravda.com.ua/cdn/covid-19/cpa/, Stand: 27.11.2020 08:17 Uhr.

Karte erstellt von der Forschungsstelle Osteuropa an der Universität Bremen in QGIS mit Geodaten von https://www.openstreetmap.org. 


\section{Covid-19-Chronik, 9. - 22. November 2020}

\begin{tabular}{|c|c|c|}
\hline Datum & $\begin{array}{l}\text { Neue Covid- } \\
\text { 19-Fälle / } \\
\text { Gesamtzahl / } \\
\text { Todesfälle* }\end{array}$ & Ereignis \\
\hline \multirow[t]{3}{*}{9.11 .2020} & $\begin{array}{c}8.687 / 469.018 \\
/ 8.565\end{array}$ & $\begin{array}{l}\text { Präsident Wolodymyr Selenskyj und der Leiter des Präsidialamtes Andrij Jermak sind posi- } \\
\text { tiv auf Covid-19 getestet worden. Sie begeben sich in Quarantäne und versprechen, ihre Ver- } \\
\text { pflichtungen auch aus der Quarantäne heraus weiter zu erfüllen. }\end{array}$ \\
\hline & & $\begin{array}{l}\text { Wegen der dramatisch angestiegenen Zahl von Covid-19-Erkrankten kündigt der leitende } \\
\text { Sanitätsarzt Wiktor Ljaschko den Übergang zur zweiten Phase der Covid-19-Pandemie an. } \\
\text { Demzufolge werden frühzeitig planbare Operationen und Hospitalisationen in ukrainischen } \\
\text { Krankenhäusern nicht mehr stattfinden. Ab jetzt werden ausschließlich Notfälle und Covid- } \\
\text { 19-Patient*innen in Krankenhäusern behandelt. }\end{array}$ \\
\hline & & $\begin{array}{l}\text { Die Belegungsrate von Krankenhausbetten mit Covid-19-Patient*innen beträgt ukraineweit } \\
62,4 \text { Prozent. Die höchste Auslastung ist in den Regionen Poltawa - 92,5 Prozent, Riwne- } \\
\text { 82,9 Prozent und Tschernihiw - 73,8 Prozent zu beobachten. In Kyjiw werden 62,4 Pro- } \\
\text { zent der Betten für die Behandlung von Covid-19-Erkrankten benötigt, gibt das ukraini- } \\
\text { sche Gesundheitsministerium bekannt. }\end{array}$ \\
\hline \multirow[t]{2}{*}{10.11 .2020} & $\begin{array}{c}10.179 / \\
479.197 / 8.756\end{array}$ & $\begin{array}{l}\text { Der ukrainische Verteidigungsminister, Andrij Taran, wurde positiv auf Covid-19 getestet, } \\
\text { informiert das Verteidigungsministerium. Darüber hinaus hat sich der ukrainische Finanz- } \\
\text { minister, Serhij Martschenko, mit dem Coronavirus infiziert. }\end{array}$ \\
\hline & & $\begin{array}{l}\text { Der Minister für die Wiedereingliederung der temporär besetzten Gebiete, Oleksij Resni- } \\
\text { kow, berichtet über die Bereitschaft der Ukraine, aufgrund der Covid-19-Pandemie huma- } \\
\text { nitäre Lager nahe der Verwaltungsgrenze zur von Russland annektierten Krim zu errichten. }\end{array}$ \\
\hline \multirow[t]{2}{*}{11.11 .2020} & $\begin{array}{c}10.611 / \\
489.808 / 8.947\end{array}$ & $\begin{array}{l}\text { Der Parlamentschef, Dmytro Rasumkow, ist positiv auf Covid-19 getestet worden. Wie } \\
\text { die anderen an Coronavirus erkrankten Beamten wird er Fernarbeit von zu Hause leisten. }\end{array}$ \\
\hline & & $\begin{array}{l}\text { Premierminister Denys Schmyhal warnt davor, dass Krankenhäuser ohne die geplante Ver- } \\
\text { schärfung der Quarantänemaßnahmen durch eine "Wochenend-Quarantäne« in einem } \\
\text { Monat überhaupt keine Patient*innen mit Covid-19 mehr werden aufnehmen können. }\end{array}$ \\
\hline \multirow[t]{3}{*}{12.11 .2020} & $\begin{array}{c}11.057 / \\
500.865 / 9.145\end{array}$ & $\begin{array}{l}\text { Erstmals werden mehr als } 11.000 \text { neue Covid-19-Erkrankungen an einem Tag diagnosti- } \\
\text { ziert. Zugleich überschreitet die Gesamtzahl der Erkrankten seit dem Beginn der Pandemie } \\
\text { eine halbe Million und die Anzahl der Opfer } 9.000 \text { Fälle. }\end{array}$ \\
\hline & & $\begin{array}{l}\text { Der Parlamentschef, Dmytro Rasumkow, und sein Stellvertreter, Ruslan Stefantschuk, sind } \\
\text { positiv auf Covid-19 getestet worden. }\end{array}$ \\
\hline & & $\begin{array}{l}\text { Laut einem UN-Bericht könnte die Ukraine die größte Rezession seit zehn Jahren erleben } \\
\text { und mehr als } 9 \text { Millionen Menschen in Armut zurücklassen. Der UN zufolge haben seit } \\
\text { Beginn der Covid-19-Pandemie mehr als } 80 \text { Prozent der Haushalte Einkommenseinbußen } \\
\text { zu beklagen und in mehr als } 40 \text { Prozent der Familien hat mindestens ein Familienmitglied } \\
\text { seinen Arbeitsplatz verloren. }\end{array}$ \\
\hline \multirow[t]{2}{*}{13.11 .2020} & $\begin{array}{c}11.787 / \\
512.652 / 9.317\end{array}$ & $\begin{array}{l}\text { Die »Wochenend-Quarantäne« tritt vorerst bis zum } 30 \text { November } 2020 \text { in Kraft. Damit ist } \\
\text { ab heute von Samstags 0:00 Uhr bis Montags 0:00 Uhr unter anderem Essen und Trinken } \\
\text { in Bars, Restaurants und Cafés verboten. Einkaufszentren, Sporthallen, Schwimmbäder und } \\
\text { weitere Einrichtungen bleiben geschlossen. Am Wochenende dürfen nur Lebensmittelge- } \\
\text { schäfte, Apotheken, öffentliche Verkehrsmittel, Tankstellen (ohne Lebensmittel-Shops) und } \\
\text { Unternehmen, die Finanz- und Renovierungsdienstleistungen anbieten, arbeiten. }\end{array}$ \\
\hline & & $\begin{array}{l}\text { Der Pressedienst der Zentralen Wahlkommission versichert, dass die von der ukrainischen } \\
\text { Regierung eingeführte »Wochenend-Quarantäne« keinen Einfluss auf die Stichwahlen der } \\
\text { Kommunalwahlen am } 15 . \text { und } 22 \text {. November haben werde. Die Stichwahlen finden unter } \\
\text { Einhaltung aller notwendigen Covid-19-Sicherheitsmaßnahmen statt. }\end{array}$ \\
\hline
\end{tabular}




\begin{tabular}{|c|c|c|}
\hline Datum & $\begin{array}{l}\text { Neue Covid- } \\
\text { 19-Fälle / } \\
\text { Gesamtzahl / } \\
\text { Todesfälle* }\end{array}$ & Ereignis \\
\hline & & $\begin{array}{l}\text { Gegen die in Kraft tretende "Wochenend-Quarantäne« wegen der Covid-19-Pandemie äußern } \\
\text { sich folgende ukrainische Städte und Regionen: Tscherkasy, Ternopil, Schytomyr, Tscherni- } \\
\text { hiw, Luzk, Lwiw, Odesa, Mykolajiw, Kramatorsk, Slawjansk, Mukatschewo. Für eine ent- } \\
\text { sprechende Petition an die Regierung wurden mehr als } 26.000 \text { Unterschriften gesammelt. } \\
\text { Somit muss sich Präsident Wolodymyr Selenskyj mit der Petition befassen. }\end{array}$ \\
\hline \multirow[t]{2}{*}{14.11 .2020} & $\begin{array}{c}12.524 / \\
525.176 / 9.508\end{array}$ & $\begin{array}{l}\text { Die an einem Tag diagnostizierte Anzahl von neuen Covid-19-Erkrankten überschreitet erst- } \\
\text { mals seit dem Beginn der Pandemie } 12.000 \text { Fälle. }\end{array}$ \\
\hline & & Gesundheitsminister Maksym Stepanow ist positiv auf Covid-19 getestet worden. \\
\hline 15.11 .2020 & $\begin{array}{c}10.681 / \\
535.857 / 9.603\end{array}$ & $\begin{array}{l}\text { Bereits am ersten Tag der Wochenend-Quarantäne erhielt die Polizei } 1.029 \text { Meldungen } \\
\text { über Verstöße gegen Covid-19-Quarantänebeschränkungen. Die meisten Verstöße wurden } \\
\text { in den Regionen Lwiw (390), Dnipropetrowsk (209), Tscherkasy (128), in der Stadt Kyjiw } \\
\text { (121) und Ternopil (74) verzeichnet. }\end{array}$ \\
\hline 16.11 .2020 & $\begin{array}{l}9.832 / 545.689 \\
/ 9.697\end{array}$ & $\begin{array}{l}\text { Der ukrainische Außenminister Dmytro Kuleba gibt bekannt, dass im Falle der Einfüh- } \\
\text { rung einer strengen Quarantäne wegen der Covid-19-Pandemie die Landesgrenzen wieder } \\
\text { geschlossen werden. Das hält er aber in naher Zukunft für unwahrscheinlich. }\end{array}$ \\
\hline \multirow[t]{2}{*}{17.11 .2020} & $\begin{array}{l}11.968 / \\
557.657 / 9.856\end{array}$ & $\begin{array}{l}\text { Premierminister Denys Schmyhal warnt vor möglichen Konsequenzen bei Nichteinhaltung } \\
\text { der Quarantäneeinschränkungen am Wochenende. In diesem Fall werde die Ukraine in } \\
\text { drei Wochen täglich } 20.000 \text { Fälle von Covid-19 verzeichnen und keine freien Plätze mehr } \\
\text { in Krankenhäusern haben. }\end{array}$ \\
\hline & & $\begin{array}{l}\text { Ein Beschluss zur Abschaffung der Wochenend-Quarantäne wegen der Covid-19-Pandemie } \\
\text { findet in der Werchowna Rada keine ausreichende Unterstützung. Lediglich } 149 \text { von not- } \\
\text { wendigen } 226 \text { Abgeordneten stimmen für die Abschaffung. }\end{array}$ \\
\hline 18.11 .2020 & $\begin{array}{l}12.496 / \\
570.153 / \\
10.112\end{array}$ & $\begin{array}{l}\text { Die Anzahl der an Covid-19 verstorbenen Personen überschreitet } 10.000 \text { Fälle seit dem } \\
\text { Beginn der Pandemie in der Ukraine. }\end{array}$ \\
\hline \multirow[t]{2}{*}{19.11 .2020} & $\begin{array}{l}13.357 / \\
583.510 / \\
10.369\end{array}$ & $\begin{array}{l}\text { Die Anzahl der neu diagnostizierten Covid-19-Fälle an einem Tag überschreitet erstmals } \\
\text { 13.000 Fälle. }\end{array}$ \\
\hline & & $\begin{array}{l}\text { Laut einer Modellierungsstudie der Kyiv School of Economics kann sich im Falle des Schei- } \\
\text { terns der Wochenend-Quarantäne die Anzahl der Todesfälle wegen der Covid-19-Pandemie } \\
\text { bis zum Ende des Jahres auf bis zu } 33.000 \text { verdreifachen. }\end{array}$ \\
\hline \multirow[t]{2}{*}{20.11 .2020} & $\begin{array}{l}14.575 / \\
598.085 / \\
10.598\end{array}$ & Erstmals werden mehr als 14.000 neue Covid-19-Fälle an einem Tag diagnostiziert. \\
\hline & & $\begin{array}{l}\text { Gesundheitsminister Maksym Stepanow teilt mit, dass ein vollständiger Lockdown in der } \\
\text { Ukraine eingeführt werden kann, sollte es täglich } 30.000 \text { neu diagnostizierte Covid-19- } \\
\text { Fälle geben. }\end{array}$ \\
\hline \multirow[t]{2}{*}{21.11 .2020} & $\begin{array}{l}14.580 / \\
612.665 / \\
10.813\end{array}$ & $\begin{array}{l}\text { Die Gesamtzahl der registrierten Covid-19-Fälle überschreitet } 600.000 \text { seit dem Beginn der } \\
\text { Pandemie in der Ukraine. }\end{array}$ \\
\hline & & $\begin{array}{l}\text { Zum 21. November sind ukraineweit } 52 \text { Prozent der für Covid-19-Patient*innen vorgesehe- } \\
\text { nen Betten belegt. Die höchste Bettenbelegung ist in den Regionen Poltawa (73,9 Prozent) } \\
\text { und Tscherkasy ( } 70,2 \text { Prozent) zu konstatieren. }\end{array}$ \\
\hline 22.11 .2020 & $\begin{array}{l}12.079 / \\
624.744 / \\
10.951\end{array}$ & $\begin{array}{l}\text { Nach der Gesamtzahl der diagnostizierten Covid-19-Fälle belegt die Ukraine den 8. Platz } \\
\text { in Europa (nach Frankreich, Russland, Spanien, Großbritannien, Italien, Deutschland und } \\
\text { Polen). }\end{array}$ \\
\hline
\end{tabular}




\section{9. -22 . November 2020}

\begin{tabular}{|c|c|}
\hline 10.11 .2020 & $\begin{array}{l}\text { Die Ukraine öffnet sieben Kontrollpunkte an der Kontaktlinie zu den »Volksrepubliken« Donezk und Luhansk, } \\
\text { nachdem diese wegen der Coronavirus-Pandemie für mehrere Wochen gesperrt waren. }\end{array}$ \\
\hline 10.11 .2020 & $\begin{array}{l}\text { Der Ausschuss für Außenpolitik im EU-Parlament begrüßt in einem neuen Bericht die Fortschritte der Ukraine } \\
\text { in Bereichen wie Landwirtschaft, Dezentralisierung und dem Bankenwesen. Gleichzeitig wird gefordert, dass } \\
\text { weitere EU-Hilfen strikt an Konditionen gebunden sein sollen und die Ukraine vor allem bei der Korruptions- } \\
\text { bekämpfung und der De-Oligarchisierung mehr unternehmen müsse. }\end{array}$ \\
\hline 11.11 .2020 & $\begin{array}{l}\text { Das Oberste Antikorruptionsgericht verurteilt einen Abgeordneten des Regionalrats von Poltawa zu einer } \\
\text { Gefängnisstrafe von } 4,5 \text { Jahren. Er hatte dem Gericht zufolge einem Polizeibeamten eine Bestechung von } \\
\text { 35.000 Hrywnja gezahlt. }\end{array}$ \\
\hline 13.11 .2020 & $\begin{array}{l}\text { Laut jüngsten Zahlen des Statistikamts der Ukraine sind die Importe von Januar bis September } 2020 \text { deut- } \\
\text { lich stärker gesunken als die Exporte, was zu einem Exportüberschuss in diesem Zeitraum von } 531 \text { Mio. US- } \\
\text { Dollar führte. Im Vorjahreszeitraum stand hingegen ein Handelsdefizit von 4,2 Mrd. US-Dollar zu Buche. } \\
\text { Ursächlich für die guten Exportzahlen sind vor allem die Ausfuhren von Gütern nach China, die um } 86 \text { Pro- } \\
\text { zent auf 4,8 Mrd. US-Dollar zunahmen. }\end{array}$ \\
\hline 15.11 .2020 & $\begin{array}{l}\text { In zahlreichen Städten und Gemeinden, in denen es in der ersten Runde der Kommunalwahlen Ende Oktober } \\
\text { keine Sieger gab, finden Stichwahlen statt, darunter in Odessa, Cherson und Luzk. Während der umstrittene } \\
\text { Bürgermeister von Odessa, Hennadij Truchanow, die Stichwahl für sich entscheiden kann, ist der Bürgermeister } \\
\text { von Kramatorsk im Osten der Ukraine der einzige Amtsinhaber in einer Großstadt, der die Stichwahl verliert. }\end{array}$ \\
\hline 16.11 .2020 & $\begin{array}{l}\text { Laut dem Statistikdienst der Ukraine wuchs die Wirtschaft im dritten Quartal } 2020 \text { um 8,5 Prozent gegen- } \\
\text { über dem zweiten Quartal, das aufgrund der Coronavirus-Pandemie um 11,4 Prozent gegenüber dem ersten } \\
\text { Quartal einbrach. }\end{array}$ \\
\hline 16.11 .2020 & $\begin{array}{l}\text { Das Kiewer Berufungsgericht hebt die Entscheidung über die »in absentia«-Festnahme des flüchtigen Ex- } \\
\text { Präsidenten Wiktor Janukowytsch wegen der gewaltsamen Auflösung der Maidan-Proteste am 18. und } 20 . \\
\text { Februar } 2014 \text { auf. Grund seien Verfahrensfehler, so ein Anwalt von Janukowytsch. Am 12. Mai } 2020 \text { hatte } \\
\text { das Petscherskyj-Bezirksgericht in Kyjiw die Inhaftierung von Janukowytsch »in absentia" angeordnet, gegen } \\
\text { die Janukowytsch in Berufung gegangen war. }\end{array}$ \\
\hline 16.11 .2020 & $\begin{array}{l}\text { Laut einem neuen Report der OSZE Sonderbeobachtungsmission für die Ukraine gab es in diesem Jahr } 18 \text { zivile } \\
\text { Todesopfer und } 85 \text { Verletzte in der Ostukraine. Aktuell sei die Lage an der Kontaktline »relativ ruhig«, so der } \\
\text { Bericht. }\end{array}$ \\
\hline 17.11 .2020 & $\begin{array}{l}\text { Nach einem Telefonat mit der IWF-Direktorin Kristalina Georgiewa teilt Präsident Wolodymyr Selenskyj mit, } \\
\text { dass der IWF die Ukraine weiter unterstützen werde, im Gegenzug jedoch die Bekämpfung der Korruption } \\
\text { und eine Lösung in der Verfassungskrise fordere. }\end{array}$ \\
\hline 18.11 .2020 & $\begin{array}{l}\text { Das Ministerkabinett ernennt nach einer längeren Überprüfung Pawel Rjabikin, seit } 2017 \text { Generaldirektor des } \\
\text { Flughafens Kyjiw-Boryspil, zum neuen Leiter des staatlichen Zolldienstes. } 2019 \text { teilte die Nationale Agentur } \\
\text { für Korruptionsprävention (NAZK) mit, dass Rjabikin } 2016 \text { Immobilien und Fahrzeuge in seinen Vermögens- } \\
\text { angaben verschleiert habe. Sein Vorgänger, Maksym Nefjodow, wurde im April } 2020 \text { im Rahmen einer grö-- } \\
\text { ßeren Entlassungswelle von Spitzenbeamten suspendiert, wogegen es viel Kritik aus dem In- und Ausland gab. } \\
\text { Nefjodow galt als erfolgreicher Reformer, dem zugetraut wurde, den notorisch korrupten Zoll zu reformierten. }\end{array}$ \\
\hline 18.11 .2020 & $\begin{array}{l}\text { Ein Sprecher der ukrainischen Delegation der Trilateralen Kontaktgruppe in Minsk teilt mit, dass noch vor } \\
\text { dem 24. Dezember ein Gefangenenaustausch zwischen der Ukraine und Russland bzw. den "Volksrepubli- } \\
\text { ken« angestrebt werde. }\end{array}$ \\
\hline 19.11 .2020 & $\begin{array}{l}\text { Bei einer Videokonferenz mit den Botschaftern der G7-Staaten versichert Präsident Wolodymyr Selenskyj, dass } \\
\text { der Direktor des Nationalen Anti-Korruptionsbüros (NABU), Artem Sytnyk, auch weiterhin Leiter der Behörde } \\
\text { bleiben werde. Hintergrund ist eine umstrittene Entscheidung des Verfassungsgerichts, das im August } 2020 \\
\text { die Entlassung Sytnyks bis Jahresende forderte, da der frühere Präsident Petro Poroschenko mit der Ernennung } \\
\text { Sytnyks seine Vollmachten überschritten hätte. Das NABU kritisierte die Entscheidung als "politisch moti- } \\
\text { viert«, da Sytnyks Behörde gegen ranghohe Richter und Politiker ermittle. }\end{array}$ \\
\hline
\end{tabular}




\begin{tabular}{|l|l|}
\hline 20.11.2020 & $\begin{array}{l}\text { Der EU-Außenbeauftragte Josip Borrell erklärt, dass sich die Ukraine gemeinsam mit sechs weiteren Staaten } \\
\text { offiziell den Sanktionen der EU gegen das Regime des selbsternannten belarussischen Machthabers Alexan- } \\
\text { der Lukaschenko angeschlossen habe. Gegen die landesweiten Proteste infolge der massiven Wahlfälschungen } \\
\text { geht das Lukaschenko-Regime gewaltsam vor, weshalb die EU am 2. Oktober Sanktionen gegen 40 Regime- } \\
\text { vertreter verhängte. Am 6. November wurden weitere 15 Personen in die Sanktionsliste aufgenommen, darun- } \\
\text { ter auch Alexander Lukaschenko. }\end{array}$ \\
\hline 20.11 .2020 & $\begin{array}{l}\text { Auf einer außerordentlichen Sitzung des Ministerkabinetts wird die amtierende Energieministerin Olha Busla- } \\
\text { wez entlassen und ihr Stellvertreter, Jurij Bojko (nicht zu verwechseln mit dem Oppositionspolitiker und frü- } \\
\text { heren stellvertretenden Ministerpräsidenten Jurij Boiko), zum Nachfolger ernannt. Der neue Energieminister } \\
\text { arbeitete vor seiner Tätigkeit als stellvertretender Energieminister in leitenden Positionen im Energiesektor, } \\
\text { darunter beim staatlichen Energieversorger »Enerhorynok». }\end{array}$ \\
\hline 22.11 .2020 & $\begin{array}{l}\text { In der Ukraine finden in 86 Städten und Gemeinden eine weitere Runde der Kommunalwahl-Stichwahlen } \\
\text { statt, darunter in Dnepr, Lwiw, Mykolajiw und anderen Großstädten. Bei den meisten Wahlen setzen sich die } \\
\text { Amtsinhaber durch, darunter Andrij Sadowyj in Lwiw und Borys Filatow in Dnipro. }\end{array}$ \\
\hline
\end{tabular}

Die Chronik wird zeitnah erstellt und basiert ausschließlich auf im Internet frei zugänglichen Quellen. Die Redaktion bemüht sich, bei jeder Meldung die ursprüngliche Quelle eindeutig zu nennen. Aufgrund der großen Zahl von manipulierten und falschen Meldungen kann die Redaktion der Ukraine-Analysen keine Gewähr für die Richtigkeit der Angaben übernehmen.

Zusammengestellt von Dr. Eduard Klein

Sie können die gesamte Chronik seit Februar 2006 auch auf http://www.laender-analysen.de/ukraine/ unter dem Link»Chronik« lesen.

Herausgeber:

Forschungsstelle Osteuropa an der Universität Bremen

Deutsche Gesellschaft für Osteuropakunde e.V. Deutsches Polen-Institut

Leibniz-Institut für Agrarentwicklung in Transformationsökonomien

Leibniz-Institut für Ost- und Südosteuropaforschung

Zentrum für Osteuropa- und internationale Studien (ZOiS) gGmbH

Redaktion:

Dr. Eduard Klein (verantwortlich)

Chronik: Dr. Eduard Klein

Satz: Matthias Neumann

Wissenschaftlicher Beirat:

Dr. Kseniia Gatskova, Leibniz-Institut für Ost- und Südosteuropaforschung Regensburg

Prof. Dr. Guido Hausmann, Leibniz-Institut für Ost- und Südosteuropaforschung Regensburg

Dr. Susan Stewart, Stiftung Wissenschaft und Politik, Berlin

Dr. Susann Worschech, Europa-Universität Viadrina, Frankfurt/0.

Die Meinungen, die in den Ukraine-Analysen geäußert werden, geben ausschließlich die Auffassung der Autoren wieder.

Abdruck und sonstige publizistische Nutzung sind nach Rücksprache mit der Redaktion gestattet.

Ukraine-Analysen-Layout: Cengiz Kibaroglu, Matthias Neumann und Michael Clemens

Alle Ausgaben der Ukraine-Analysen sind mit Themen- und Autorenindex archiviert unter www laender-analysen de

Die Ukraine-Analysen werden im Rahmen eines Lizenzvertrages in das Internetangebot der Bundeszentrale für politische Bildung (www.bpb.de) aufgenommen.

ISSN 1862-555X ๔ 2020 by Forschungsstelle Osteuropa an der Universität Bremen, Deutsche Gesellschaft für Osteuropakunde e.V., Deutsches Polen-Institut, Leibniz-Institut für Agrarentwicklung in Transformationsökonomien, Leibniz-Institut für Ost- und Südosteuropaforschung, Zentrum für Osteuropa- und internationale Studien (Z0iS) gGmbH Forschungsstelle Osteuropa • Länder-Analysen • Klagenfurter Str. 8 • 28359 Bremen • Telefon: + 49 421-218-69600 • Telefax: +49 421-218-69607 e-mail: laender-analysen@uni-bremen.de•Internet-Adresse: http://www.laender-analysen.de/ukraine/ 


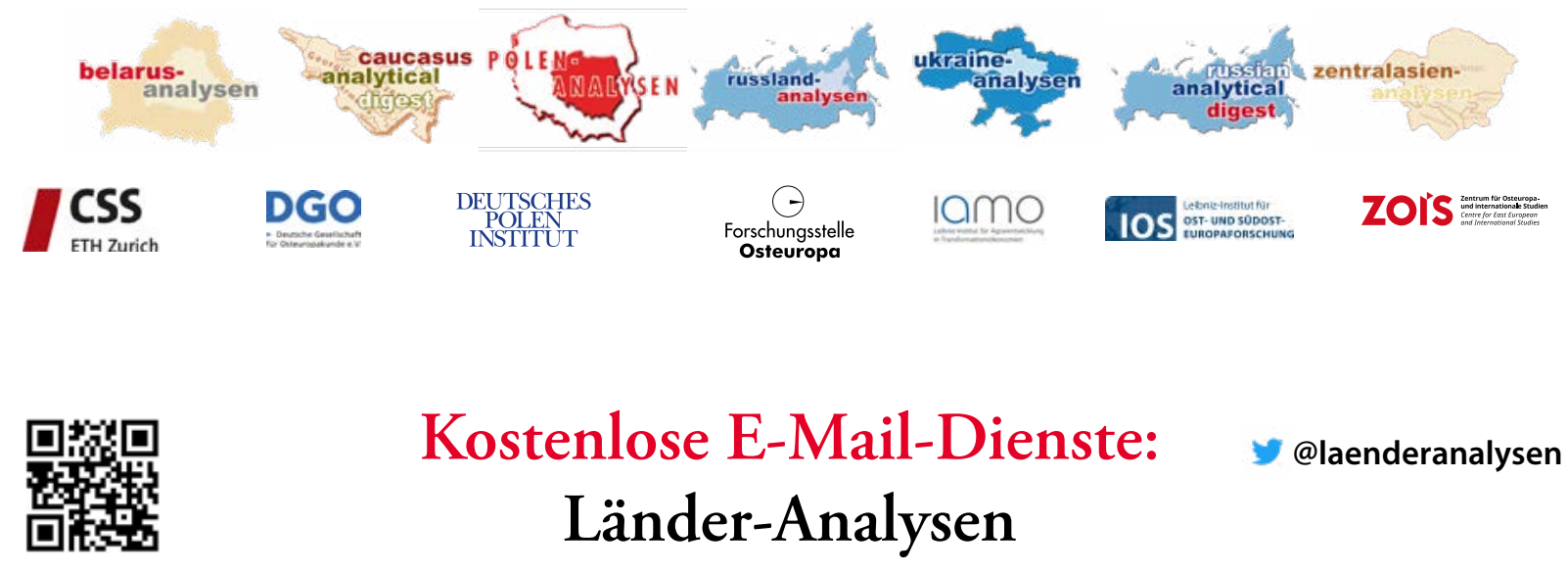

Die Länder-Analysen bieten regelmäßig im kostenlosen Abonnement kompetente Einschätzungen aktueller politischer, wirtschaftlicher, sozialer und kultureller Entwicklungen in Ostmitteleuropa und der GUS. Alle Länder-Analysen verstehen sich als Teil eines gemeinsamen Projektes, das der wissenschaftlich fundierten, allgemeinverständlich formulierten Analyse der Entwicklungen im östlichen Europa, der Offenheit für verschiedene inhaltliche Positionen und der kostenlosen und nicht-kommerziellen Information einer breit verstandenen interessierten Öffentlichkeit verpflichtet ist. Autor/innen sind internationale Fachwissenschaftler/innen und Expert/innen. Die Redaktionen der Länder-Analysen bestehen aus Wissenschaftler/innen mit langjähriger Forschungserfahrung.

Die deutschsprachigen Länder-Analysen werden gemeinsam von der Forschungsstelle Osteuropa an der Universität Bremen, dem Zentrum für Osteuropa- und internationale Studien, der Deutschen Gesellschaft für Osteuropakunde, dem Deutschen Polen-Institut, dem Leibniz-Institut für Agrarentwicklung in Transformationsökonomien und dem Leibniz-Institut für Ost- und Südosteuropaforschung herausgegeben. Die englischsprachigen Länder-Analysen erscheinen in Kooperation der Forschungsstelle Osteuropa mit dem Center for Security Studies (CSS) der ETH Zürich.

Die Länder-Analysen bieten regelmäßig Kurzanalysen zu aktuellen Themen, ergänzt um Grafiken und Tabellen sowie Dokumentationen. Zusätzlich gibt es eine Chronik aktueller Ereignisse.

\section{Belarus-Analysen}

Erscheinungsweise: zweimonatlich

Abonnement unter: http://www.laender-analysen.de/belarus/

\section{Caucasus Analytical Digest}

In englischer Sprache. Erscheinungsweise: zweimonatlich

Abonnement unter: http://www.css.ethz.ch/en/publications/cad.html

\section{Polen-Analysen}

Erscheinungsweise: zweimal monatlich

Abonnement unter: http://www.deutsches-polen-institut.de/newsletter/polen-analysen/

\section{Russland-Analysen}

Erscheinungsweise: zweimal monatlich

Abonnement unter: http://www.laender-analysen.de/russland/

\section{Russian Analytical Digest}

In englischer Sprache. Erscheinungsweise: zweimal monatlich

Abonnement unter: http://www.css.ethz.ch/en/publications/rad.html

\section{Ukraine-Analysen}

Erscheinungsweise: zweimal monatlich

Abonnement unter: http://www.laender-analysen.de/ukraine/

\section{Zentralasien-Analysen}

Erscheinungsweise: zweimonatlich

Abonnement unter: http://www.laender-analysen.de/zentralasien/ 